\title{
Association of Glutathione S-transferase gene polymorphism with bladder Cancer susceptibility
}

Tianbiao Zhou ${ }^{1 *+} \mathbb{D}$, Hong-Yan Li ${ }^{2 \dagger}$, Wei-Ji Xie ${ }^{1}$, Zhiqing Zhong ${ }^{1}$, Hongzhen Zhong ${ }^{1}$ and Zhi-Jun Lin ${ }^{1}$

\begin{abstract}
Background: We conducted a meta-analysis to evaluate the relationship between the glutathione S-transferase $\mu 1$ (GSTM1) - and glutathione S-transferase $\theta 1$ (GSTT1) - null genotypes and susceptibility to bladder cancer.

Methods: We identified association reports from the databases of PubMed, Embase, the Cochrane Library and the China Biological Medicine Database (CBM disc) on July 1, 2017 and synthesized eligible investigations. Results were expressed using odds ratios (ORs) for dichotomous data, and we also calculated $95 \%$ confidence intervals (Cls).

Results: In this meta-analysis, we found that the GSTM1-null genotype was associated with bladder cancer risk in the overall population, and individually in whites, Africans and Asians (overall population: $\mathrm{OR}=1.40,95 \% \mathrm{Cl}$ : $1.31-$ 1.48, $P<0.00001$; whites: $\mathrm{OR}=1.39,95 \%$ Cl: 1.26-1.54, $P<0.00001$; Africans: $\mathrm{OR}=1.54,95 \% \mathrm{Cl}: 1.16-2.05, P=0.003$; Asians: $\mathrm{OR}=1.45,95 \% \mathrm{Cl}: 1.33-1.59, P<0.00001)$. The GSTT1-null genotype was associated with bladder cancer risk in the overall population, but not in whites, in Africans or Asians (overall population: $\mathrm{OR}=1.11,95 \% \mathrm{Cl}: 1.01-1.22, P=$ 0.03; whites: $O R=1.16,95 \% \mathrm{Cl}: 0.99-1.36, P=0.07$; Africans: $\mathrm{OR}=1.07,95 \% \mathrm{Cl}: 0.65-1.76, P=0.79$; Asians: $\mathrm{OR}=1.05$, 95\% Cl: 0.91-1.22, $P=0.51)$. Interestingly, a dual-null GSTM1-GST11 genotype was associated with bladder cancer risk in the overall population and in Asians (overall population: $\mathrm{OR}=1.48,95 \% \mathrm{Cl}: 1.15-1.92, P=0.002$; Asians: $\mathrm{OR}=$ 1.62, 95\% Cl: 1.15-2.28, $P=0.006$ ). In conclusion, the GSTM1-null, GST11-null and dual-null GSTM1-GST11 genotypes might be associated with the onset of bladder cancer, but additional genetic-epidemiological studies should be conducted to explore this association further.
\end{abstract}

Keywords: Bladder cancer, Gene polymorphism, GSTM1, GSTT1, GSTP1, Meta-analysis

\section{Background}

Bladder cancer, also known as urothelial cancer of the bladder, is the most common malignancy affecting the urinary system [1-3]. Treatment of bladder cancer has not advanced in the past 30 years [1]. The disease has a multifactorial etiology that includes environmental factors such as cigarette smoking, arsenic exposure and occupational exposure as well as genetic factors [4-6]. Genetic factors are the one of the most important factors associated with the onset of bladder cancer [7]. Smoking is a major risk factor for the development of this cancer, but the functional consequences of the carcinogens in tobacco

\footnotetext{
* Correspondence: zhoutb@aliyun.com

†Tianbiao Zhou and Hongyan Li contributed equally to this work.

'Department of Nephrology, The Second Affiliated Hospital of Shantou

University Medical College, Shantou 515041, China

Full list of author information is available at the end of the article
}

smoke in terms of bladder cancer-associated metabolic changes remain poorly defined. Current evidence indicates that some gene polymorphisms are associated with bladder cancer morbidity [8-12].

Glutathione S-transferases (GSTs) play an important role in detoxification of various toxic compounds, such as carcinogens, and are a family of enzymes that include the glutathione S-transferase $\mu 1$ (GSTM1), $\theta 1$ (GSTT1) and $\pi 1$ (GSTP1) classes, etc. [13]. They are important phase II detoxifying enzymes that catalyze the conjugation of reduced glutathione (GSH) to hydrophobic, electrophilic xenobiotic substances [14]. Genetic risk to malignant tumors has led to the accumulating attention to the investigations of genes polymorphism involved in process of carcinogenesis [15]. The gene polymorphisms of GSTs might influence the detoxification activities of the enzymes, predisposing individuals to cancers, such

(c) The Author(s). 2018 Open Access This article is distributed under the terms of the Creative Commons Attribution 4.0 International License (http://creativecommons.org/licenses/by/4.0/), which permits unrestricted use, distribution, and 
as oral squamous-cell carcinoma, gynecological cancer, breast cancer, prostate cancer, hepatocellular carcinoma, and colorectal cancer [16-21].

In the past few decades, most of the epidemiological investigations have focused on the relationship between the null genotypes for GSTM1-GSTT1 and bladder cancer susceptibility. However, available evidence is inadequate due to the sparseness of data or disagreements among reported studies. We performed this meta-analysis to investigate whether the dual-null GSTM1-GSTT1 genotype was associated with bladder cancer susceptibility.

\section{Methods}

\section{Search strategy}

We retrieved relevant published articles from the electronic databases of PubMed, Embase, the Cochrane Library and the China Biological Medicine Database (CBM-disc) on July 1, 2017, and we recruited eligible original articles for our meta-analysis. Key search terms consisted of ["glutathione S-transferases” OR “GSTs” OR "GSTM1” OR "GSTT1"] and ["bladder cancers" OR "bladder cancer"]. We identified additional articles through references cited in retrieved articles, and we also examined citations of retrieved articles and the previous meta-analyses.

\section{Inclusion and exclusion criteria Inclusion criteria}

(1) The endpoint of each study had to be bladder cancers. (2) The study had to include 2 comparison groups (bladder cancers vs. controls). (3) The study had to provide detailed data on genotype distribution.

\section{Exclusion criteria}

(1) Case reports, review articles and editorials. (1) Preliminary results not focused on GSTM1, GSTT1 or outcome. (3) Investigating the relationship of GST gene expression to disease. (4) Multiple publications.

\section{Quality appraisal}

To evaluate the quality of the recruited articles that met the above-listed inclusion criteria, we used a quality score based on 7 aspects of genetic-association studies (Additional file 1: Table S1). Thakkinstian et al. [22] created the quality score form in 2005; its range spans from 0 (worst quality) to 12 (best quality). Two researchers who were responsible for literature retrieval appraised quality independent of one another, and a discussion was made until every respect was entirely consistent by comparison.

\section{Data extraction and synthesis}

Two investigators independently excerpted the following information from each eligible study: first author's surname, year of publication, and number of cases and controls for both the GSTM1 and GSTT1 genotypes. We calculated frequencies for both the disease group and the control group from the corresponding genotype distribution. Finally, we compared the results and resolved any disagreements by discussion. We tested the consistency of the data extracted by the 2 researchers, and any disagreement was again resolved by discussion.

\section{Statistical analysis}

We performed all statistical analyses using Cochrane Review Manager Software, version 5 (RevMan 5; Cochrane Library, UK). We used $I^{2}$ to test heterogeneity among the included studies, and we counted the pooled statistic using a fixed-effects model (Cochran-Mantel-Haenszel method), but switched to a random-effects model (DerSimonian-Laird method) when the $P$-value of the heterogeneity test was $<0.1$. Results were expressed with odds ratios (ORs) for dichotomous data, and we also calculated 95\% confidence intervals (CIs). $P<0.05$ was required for the pooled OR to be statistically significant. We graphically judged publication bias from the Begg adjusted-rank correlation test [23] and the Egger regression asymmetry test [24] using the Stata version 12.0 (Stata Corporation, College Station, TX), and $P$-values < 0.1 were considered significant.

\section{Results}

Study characteristics for the GSTM1-null genotype and bladder cancer risk

We included 72 studies [25-96], which contained 20,239 case series and 24,393 controls, in our assessment of the relationship between the GSTM1-null genotype and bladder cancer susceptibility (Fig. 1 and Table 1). We extracted data of interest: first author's surname, year of publication and number of cases and controls for the GSTM1-null genotype (Table 1). Average distribution frequency of the GSTM1-null genotype was $56.15 \%$ in the bladder cancer group and $46.97 \%$ in the control group, indicating that the GSTM1-null genotype was higher in the bladder cancer cases than in the controls (case/control $=1.20$ ).

In the subgroup of patients and controls who smoked cigarettes, we included 24 studies [25, 30, 34, 35, 42, 43, 47, 48, $50,51,54-56,64,65,68,69,73,76,83,85,91,92,95]$ (data not shown) containing 3724 case series and 3160 controls. Average distribution frequency of the GSTM1-null genotype was $55.67 \%$ in the bladder cancer group and $47.57 \%$ in the control group, indicating that the GSTM1-null genotype was significantly higher in the bladder cancer cases compared with the controls (case/control $=1.17$ ).

\section{Study characteristics for GSTT1-null genotype and bladder cancer risk}

We included 61 studies [29, 32-34, 36-40, 42-45, 4753, 55-61, 63-69, 72-74, 76, 77, 79, 83-86, 89, 91, 92, 95-108] containing 13,041 case series and 16,739 


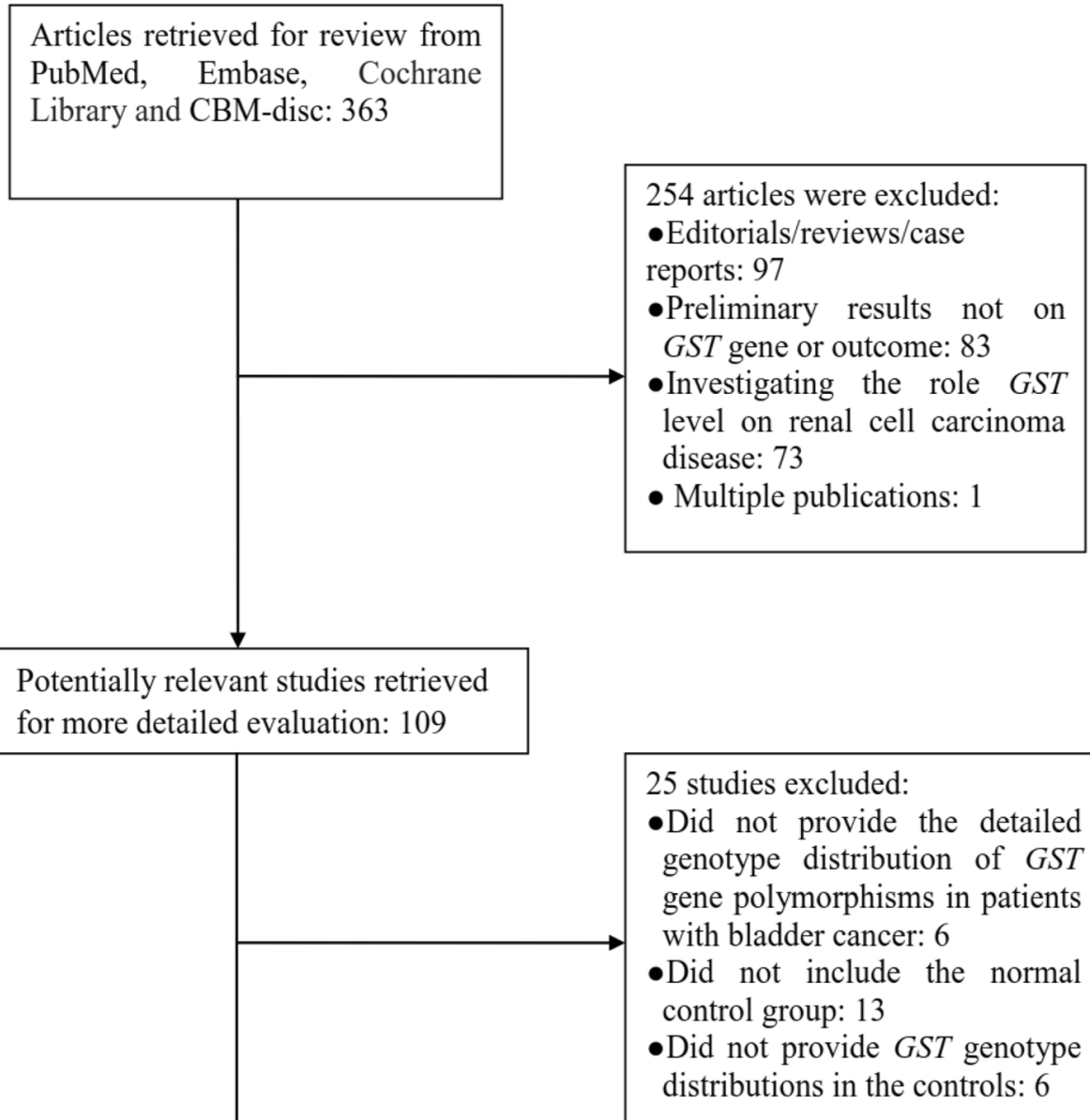

Studies included in the meta-analysis: 84

GSTM1: 72

GSTT1: 61

GSTM1-GSTT1: 18

Fig. 1 Flow chart of the study search and selection

controls in our assessment of the relationship between the GSTT1-null genotype and bladder cancer risk (Fig. 1 and Table 2). Average distribution frequency of the GSTT1-null genotype was $29.58 \%$ in the bladder cancer group and $26.67 \%$ in the control group, indicating that the GSTT1 -null genotype was higher in the bladder cancer cases compared with the controls (case/control =1.11).

In the subgroup of patients and controls who smoked cigarettes, we included 21 studies [34, 42, 43, 47, 48, 50, $51,55,56,64,65,68,69,73,76,83,85,91,92,95,97]$ (data not shown) containing 3170 case series and 2793 controls. Average distribution frequency of the GSTT1-null genotype was $29.29 \%$ in the bladder cancer group and $28.65 \%$ in the control group-that is, similar in both groups (case/control $=1.02$ ).

\section{Study characteristics for the dual-null GSTM1-GSTT1 genotype and bladder cancer risk}

We included 18 studies [32, 37, 39, 43, 47, 48, 52, 55, 58, $60,63,65,67,79,84,85,89,96]$ containing 2426 case series and 3874 controls in our assessment of the relationship between the dual-null GSTM1-GSTT1 genotype and bladder cancer risk (Fig. 1 and Table 3). Average distribution frequency of the dual-null GSTM1-GSTT1 genotype was $16.78 \%$ in the bladder cancer group and $11.45 \%$ in the control group. Therefore, the dual-null GSTM1-GSTT1 genotype was 
Table 1 Characteristics of the studies evaluating effects of the GSTM1-null genotypes on bladder carcinogen risk

\begin{tabular}{|c|c|c|c|c|c|c|c|c|c|c|}
\hline \multirow[t]{2}{*}{ Author, year } & \multirow[t]{2}{*}{ Country } & \multirow[t]{2}{*}{ Ethnicity } & \multirow{2}{*}{$\begin{array}{l}\text { Source of } \\
\text { controls }\end{array}$} & \multirow{2}{*}{$\begin{array}{l}\text { Quality } \\
\text { Score }\end{array}$} & \multicolumn{3}{|l|}{ Case } & \multicolumn{3}{|c|}{ Control } \\
\hline & & & & & - & + & total & - & + & total \\
\hline \multirow[t]{3}{*}{ Bell 1993} & \multirow[t]{3}{*}{ USA } & Overall & Population-based & \multirow[t]{3}{*}{9} & 111 & 89 & 200 & 85 & 115 & 200 \\
\hline & & Caucasian & Population-based & & 61 & 39 & 100 & 50 & 50 & 100 \\
\hline & & African & Population-based & & 50 & 50 & 100 & 35 & 65 & 100 \\
\hline Daly 1993 & UK & Caucasian & Population-based & 4 & 45 & 8 & 53 & 31 & 27 & 58 \\
\hline Zhong 1993 & UK & Caucasian & Hospital-based & 4 & 39 & 58 & 97 & 94 & 131 & 225 \\
\hline \multirow[t]{4}{*}{ Lin 1994} & \multirow[t]{4}{*}{ USA, etc } & Overall & Population-based & \multirow[t]{4}{*}{6} & 61 & 46 & 107 & 442 & 473 & 915 \\
\hline & & Caucasian & Population-based & & 52 & 37 & 89 & 236 & 243 & 479 \\
\hline & & Asian & Population-based & & 5 & 1 & 6 & 179 & 170 & 349 \\
\hline & & African & Population-based & & 4 & 8 & 12 & 27 & 60 & 87 \\
\hline Okkels 1996 & Denmark & Caucasian & Hospital-based & 7 & 133 & 100 & 233 & 100 & 100 & 200 \\
\hline Anwar 1996 & Egypt & African & Population-based & 9 & 19 & 3 & 22 & 10 & 11 & 21 \\
\hline Brockmoller 1996 & Germany & Caucasian & Hospital-based & 8 & 217 & 157 & 374 & 192 & 181 & 373 \\
\hline Lafuente 1996 & Egypt & African & Population-based & 5 & 39 & 27 & 66 & 28 & 27 & 55 \\
\hline Katoh 1998 & Japan & Asian & Hospital-based & 9 & 66 & 46 & 112 & 50 & 62 & 112 \\
\hline Abdel-Rahman 1998 & Egypt & African & Hospital-based & 8 & 26 & 11 & 37 & 15 & 19 & 34 \\
\hline Salagovic 1999 & Slovakia & Caucasian & Hospital-based & 6 & 40 & 36 & 76 & 123 & 125 & 248 \\
\hline Mungan 2000 & Netherlands & Caucasian & Hospital-based & 4 & 38 & 23 & 61 & 30 & 39 & 69 \\
\hline Peluso 2000 & Italy & Caucasian & Hospital-based & 5 & 61 & 69 & 130 & 29 & 25 & 54 \\
\hline Schnakenberg 2000 & Germany & Caucasian & Population-based & 6 & 93 & 64 & 157 & 129 & 94 & 223 \\
\hline Steinhoff 2000 & Germany & Caucasian & Hospital-based & 7 & 80 & 55 & 135 & 57 & 70 & 127 \\
\hline Georgiou 2000 & Greece & Caucasian & Hospital-based & 6 & 56 & 33 & 89 & 56 & 91 & 147 \\
\hline Kim 2000 & Korea & Asian & Hospital-based & 6 & 78 & 34 & 112 & 128 & 97 & 225 \\
\hline Toruner 2001 & Turkey & Asian & Hospital-based & 8 & 75 & 46 & 121 & 55 & 66 & 121 \\
\hline Aktas 2001 & Turkey & Asian & Population-based & 4 & 56 & 47 & 103 & 70 & 132 & 202 \\
\hline Giannakopoulos 2002 & Greece & Caucasian & Hospital-based & 6 & 56 & 33 & 89 & 56 & 91 & 147 \\
\hline Kim 2002 & Korea & Asian & Population-based & 8 & 138 & 78 & 216 & 265 & 184 & 449 \\
\hline Lee 2002 & Korea & Asian & Hospital-based & 8 & 149 & 83 & 232 & 86 & 79 & 165 \\
\hline Ma 2002 & China & Asian & Population-based & 8 & 180 & 137 & 317 & 99 & 83 & 182 \\
\hline Schroeder 2003 & USA & Mix & Hospital-based & 8 & 137 & 93 & 230 & 101 & 112 & 213 \\
\hline Jong 2003 & Korea & Asian & Population-based & 9 & 75 & 51 & 126 & 99 & 105 & 204 \\
\hline Moore 2004 & USA & Mix & Population-based & 8 & 54 & 52 & 106 & 49 & 60 & 109 \\
\hline Srivastava 2004 & India & Asian & Hospital-based & 7 & 42 & 64 & 106 & 54 & 128 & 182 \\
\hline Hung 2004 & France & Caucasian & Hospital-based & 7 & 132 & 69 & 201 & 112 & 102 & 214 \\
\hline Saad 2005 & UK & Caucasian & Population-based & 8 & 45 & 27 & 72 & 40 & 41 & 81 \\
\hline Srivastava 2005 & India & Asian & Population-based & 10 & 140 & 230 & 370 & 43 & 63 & 106 \\
\hline Sobti 2005 & India & Asian & Population-based & 9 & 37 & 63 & 100 & 24 & 52 & 76 \\
\hline Garcia-Closas 2005 & Spain & Caucasian & Hospital-based & 9 & 716 & 422 & 1138 & 571 & 561 & 1132 \\
\hline Karagas 2005 & USA & Mix & Population-based & 9 & 210 & 134 & 344 & 309 & 233 & 542 \\
\hline Kim 2005 & Korea & Asian & Hospital-based & 7 & 92 & 61 & 153 & 73 & 80 & 153 \\
\hline McGrath 2006 & USA & Mix & Population-based & 11 & 109 & 82 & 191 & 483 & 439 & 922 \\
\hline Ouerhani 2006 & Tunisia & African & Population-based & 6 & 39 & 23 & 62 & 36 & 43 & 79 \\
\hline Murta-Nascimento 2007 & Spain & Caucasian & Hospital-based & 8 & 428 & 251 & 679 & 367 & 368 & 735 \\
\hline Moore 2007 & Spain & Caucasian & Hospital-based & 7 & 683 & 394 & 1077 & 524 & 498 & 1022 \\
\hline
\end{tabular}


Table 1 Characteristics of the studies evaluating effects of the GSTM1-null genotypes on bladder carcinogen risk (Continued)

\begin{tabular}{|c|c|c|c|c|c|c|c|c|c|c|}
\hline \multirow[t]{2}{*}{ Author, year } & \multirow[t]{2}{*}{ Country } & \multirow[t]{2}{*}{ Ethnicity } & \multirow{2}{*}{$\begin{array}{l}\text { Source of } \\
\text { controls }\end{array}$} & \multirow{2}{*}{$\begin{array}{l}\text { Quality } \\
\text { Score }\end{array}$} & \multicolumn{3}{|l|}{ Case } & \multicolumn{3}{|c|}{ Control } \\
\hline & & & & & - & + & total & - & + & total \\
\hline Cengiz 2007 & Turkey & Caucasian & Hospital-based & 6 & 34 & 17 & 51 & 22 & 31 & 53 \\
\hline Kellen 2007 & Belgium & Caucasian & Population-based & 8 & 312 & 267 & 579 & 597 & 466 & 1063 \\
\hline Zhao 2007 & USA & Caucasian & Hospital-based & 8 & 324 & 298 & 622 & 317 & 316 & 633 \\
\hline Shao 2008 & China & Asian & Hospital-based & 10 & 85 & 117 & 202 & 81 & 191 & 272 \\
\hline Yuan 2008 & USA & Mix & Population-based & 11 & 387 & 275 & 662 & 335 & 351 & 686 \\
\hline Covolo 2008 & Italy & Caucasian & Hospital-based & 7 & 128 & 69 & 197 & 111 & 100 & 211 \\
\hline Golka 2008 & Germany & Caucasian & Hospital-based & 7 & 184 & 109 & 293 & 88 & 88 & 176 \\
\hline Song 2009 & China & Asian & Hospital-based & 11 & 131 & 77 & 208 & 108 & 104 & 212 \\
\hline Altayli 2009 & Turkey & Caucasian & Hospital-based & 7 & 58 & 77 & 135 & 65 & 63 & 128 \\
\hline Grando 2009 & Brazil & Mix & Population-based & 7 & 40 & 60 & 100 & 33 & 67 & 100 \\
\hline Lin 2009 & USA & Mix & Population-based & 9 & 312 & 292 & 604 & 286 & 324 & 610 \\
\hline Zupa 2009 & Italy & Caucasian & Population-based & 8 & 13 & 10 & 23 & 68 & 53 & 121 \\
\hline Abd 2010 & Egypt & African & Hospital-based & 6 & 11 & 9 & 20 & 9 & 11 & 20 \\
\hline Moore 2011 & USA & Mix & Hospital-based & 10 & 653 & 400 & 1053 & 690 & 545 & 1235 \\
\hline Öztürk 2011 & Turkey & Caucasian & Population-based & 8 & 98 & 78 & 176 & 51 & 46 & 97 \\
\hline Rouissi 2011 & Tunisia & African & Population-based & 7 & 63 & 62 & 125 & 56 & 69 & 125 \\
\hline Salinas-Sonchez 2011 & Spain & Caucasian & Hospital-based & 5 & 109 & 92 & 201 & 78 & 115 & 193 \\
\hline Goerlitz 2011 & Egypt & African & Hospital-based & 9 & 344 & 274 & 618 & 332 & 289 & 621 \\
\hline Marenne 2012 & Spain & Caucasian & Hospital-based & 7 & 488 & 285 & 773 & 402 & 357 & 759 \\
\hline Ovsiannikov 2012 & Germany & Caucasian & Hospital-based & 6 & 102 & 94 & 196 & 123 & 112 & 235 \\
\hline Schwender 2012 & Germany & Caucasian & Hospital-based & 7 & 909 & 663 & 1572 & 863 & 876 & 1739 \\
\hline Henriquez-Hernondez 2012 & Spain & Caucasian & Hospital-based & 8 & 23 & 67 & 90 & 17 & 64 & 81 \\
\hline Lesseur 2012 & New Hampshire & Caucasian & Hospital-based & 9 & 378 & 275 & 653 & 508 & 420 & 928 \\
\hline Zhang 2012 & USA & Mix & Hospital-based & 10 & 381 & 329 & 710 & 402 & 380 & 782 \\
\hline Matic 2013 & Serbia & Caucasian & Hospital-based & 8 & 111 & 90 & 201 & 61 & 61 & 122 \\
\hline Savic-Radojevic 2013 & Serbia & Caucasian & Hospital-based & 6 & 45 & 35 & 80 & 32 & 28 & 60 \\
\hline Safarinejad 2013 & Iran & Asian & Hospital-based & 10 & 50 & 116 & 166 & 93 & 239 & 332 \\
\hline Wang 2013 & China & Asian & Hospital-based & 7 & 699 & 351 & 1050 & 834 & 570 & 1404 \\
\hline Berber 2013 & Turkey & Caucasian & Hospital-based & 7 & 54 & 60 & 114 & 51 & 63 & 114 \\
\hline Kang 2013 & Korea & Asian & Hospital-based & 9 & 65 & 45 & 110 & 103 & 117 & 220 \\
\hline Reszka 2014 & Poland & Caucasian & Population-based & 9 & 149 & 95 & 244 & 165 & 200 & 365 \\
\hline Ceylan 2015 & Turkey & Caucasian & Hospital-based & 8 & 22 & 43 & 65 & 31 & 39 & 70 \\
\hline Elhawary 2017 & Saudi Arabia & Asian & Hospital-based & 7 & 24 & 28 & 52 & 40 & 64 & 104 \\
\hline Ali 2017 & Pakistan & Asian & Population-based & 11 & 83 & 117 & 200 & 57 & 143 & 200 \\
\hline
\end{tabular}

significantly higher in the bladder cancer cases compared with the controls (case/control $=1.47)$.

\section{Association of the GSTM1-null genotype with bladder cancer risk}

In this meta-analysis, we found that the GSTM1-null genotype was associated with bladder cancer risk in the overall population, and individually in whites, Africans and Asians (overall population: $\mathrm{OR}=1.40,95 \% \mathrm{CI}$ : $1.31-$
1.48, $P<0.00001$; whites: $\mathrm{OR}=1.39,95 \% \mathrm{CI}: 1.26-1.54$, $P<0.00001$; Africans: $\mathrm{OR}=1.54,95 \% \mathrm{CI}: 1.16-2.05, P=$ 0.003; Asians: $\mathrm{OR}=1.45$, 95\% CI: $1.33-1.59, P<0.00001$ ); as well as in controls from both hospital-based and population-based studies that included both high- and low-quality studies (Fig. 2 for the overall population; Table 4). In the meta-analysis for all patients and controls who smoked cigarettes, we found that the GSTM1-null genotype was associated with bladder 
Table 2 Characteristics of the studies evaluating effects of the GSTT1-null genotype of on bladder carcinogen risk

\begin{tabular}{|c|c|c|c|c|c|c|c|c|c|c|}
\hline \multirow[t]{2}{*}{ Author, Year } & \multirow[t]{2}{*}{ Country } & \multirow[t]{2}{*}{ Ethnicity } & \multirow{2}{*}{$\begin{array}{l}\text { Source of } \\
\text { controls }\end{array}$} & \multirow{2}{*}{$\begin{array}{l}\text { Quality } \\
\text { score }\end{array}$} & \multicolumn{3}{|l|}{ Case } & \multicolumn{3}{|c|}{ Control } \\
\hline & & & & & - & + & total & - & + & total \\
\hline Brockmoller 1996 & Germany & Caucasian & Hospital-based & 8 & 66 & 308 & 374 & 78 & 295 & 373 \\
\hline Kempkes 1996 & Germany & Caucasian & Population-based & 7 & 20 & 93 & 113 & 31 & 139 & 170 \\
\hline Abdel-Rahman 1998 & Egypt & African & Hospital-based & 8 & 17 & 20 & 37 & 5 & 29 & 34 \\
\hline Katoh 1998 & Japan & Caucasian & Hospital-based & 9 & 46 & 66 & 112 & 59 & 53 & 112 \\
\hline Kim 1998 & Korea & Asian & Hospital-based & 7 & 18 & 49 & 67 & 29 & 38 & 67 \\
\hline Lee 1999 & Korea & Asian & Hospital-based & 7 & 93 & 65 & 158 & 66 & 65 & 131 \\
\hline Salagovic 1999 & Slovakia & Caucasian & Hospital-based & 6 & 21 & 55 & 76 & 42 & 206 & 248 \\
\hline Georgiou 2000 & Greece & Caucasian & Hospital-based & 6 & 5 & 84 & 89 & 16 & 131 & 147 \\
\hline Peluso 2000 & Italy & Caucasian & Hospital-based & 5 & 14 & 108 & 122 & 6 & 48 & 54 \\
\hline Kim 2000 & Korea & Asian & Hospital-based & 6 & 47 & 65 & 112 & 101 & 119 & 220 \\
\hline Steinhoff 2000 & Germany & Caucasian & Hospital-based & 7 & 20 & 115 & 135 & 17 & 110 & 127 \\
\hline Schnakenberg 2000 & Germany & Asian & Hospital-based & 6 & 28 & 129 & 157 & 48 & 175 & 223 \\
\hline Toruner 2001 & Turkey & Asian & Hospital-based & 8 & 24 & 97 & 121 & 21 & 100 & 121 \\
\hline Giannakopoulos 2002 & Greece & Caucasian & Hospital-based & 6 & 5 & 84 & 89 & 16 & 131 & 147 \\
\hline Lee 2002 & Korea & Asian & Hospital-based & 8 & 135 & 97 & 232 & 85 & 80 & 165 \\
\hline Ma 2002 & China & Asian & Population-based & 8 & 29 & 32 & 61 & 88 & 94 & 182 \\
\hline Kim 2002 & Korea & Asian & Population-based & 8 & 91 & 125 & 216 & 228 & 221 & 449 \\
\hline Gago-Dominguez 2003 & USA & Mix & Population-based & 8 & 50 & 146 & 196 & 34 & 142 & 176 \\
\hline Jong 2003 & Korea & Asian & Hospital-based & 9 & 68 & 58 & 126 & 113 & 91 & 204 \\
\hline Chen 2004 & China & Asian & Population-based & 8 & 32 & 30 & 62 & 51 & 30 & 81 \\
\hline Moore 2004 & USA & Mix & Population-based & 8 & 17 & 89 & 106 & 12 & 97 & 109 \\
\hline Hung 2004 & France & Caucasian & Hospital-based & 7 & 43 & 158 & 201 & 33 & 181 & 214 \\
\hline Srivastava 2004 & India & Asian & Hospital-based & 7 & 28 & 78 & 106 & 29 & 153 & 182 \\
\hline Sanyal 2004 & Sweden & Caucasian & Population-based & 8 & 66 & 204 & 270 & 12 & 110 & 122 \\
\hline Broberg 2005 & Sweden & Caucasian & Population-based & 9 & 7 & 54 & 61 & 22 & 132 & 154 \\
\hline Garcia-Closas 2005 & Spain & Caucasian & Hospital-based & 9 & 230 & 899 & 1129 & 248 & 873 & 1121 \\
\hline Saad 2005 & UK & Caucasian & Population-based & 8 & 26 & 46 & 72 & 14 & 67 & 81 \\
\hline Karagas 2005 & USA & Mix & Population-based & 9 & 53 & 83 & 136 & 301 & 458 & 759 \\
\hline Golka 2005 & Dortmund & Caucasian & Hospital-based & 8 & 30 & 106 & 136 & 38 & 125 & 163 \\
\hline Kim 2005 & Korea & Asian & Hospital-based & 7 & 71 & 82 & 153 & 89 & 64 & 153 \\
\hline Srivastava 2005 & India & Asian & Population-based & 10 & 28 & 78 & 106 & 79 & 291 & 370 \\
\hline Shao 2005 & China & Asian & Population-based & 7 & 204 & 201 & 405 & 195 & 194 & 389 \\
\hline Sobti 2005 & India & Asian & Population-based & 9 & 30 & 70 & 100 & 11 & 65 & 76 \\
\hline McGrath 2006 & USA & Mix & Population-based & 11 & 35 & 156 & 191 & 148 & 776 & 924 \\
\hline Ouerhani 2006 & Tunisia & African & Population-based & 6 & 26 & 36 & 62 & 35 & 44 & 79 \\
\hline Kogevinas 2006 & Spain & Caucasian & Hospital-based & 8 & 24 & 75 & 99 & 17 & 74 & 91 \\
\hline Cengiz 2007 & Turkey & Caucasian & Hospital-based & 6 & 18 & 33 & 51 & 11 & 42 & 53 \\
\hline Kellen 2007 & Belgium & Caucasian & Population-based & 8 & 30 & 164 & 194 & 61 & 319 & 380 \\
\hline Zhao 2007 & USA & Caucasian & Hospital-based & 8 & 103 & 520 & 623 & 115 & 519 & 634 \\
\hline Covolo 2008 & Italy & Caucasian & Hospital-based & 7 & 42 & 155 & 197 & 33 & 178 & 211 \\
\hline Yuan 2008 & USA & Mix & Population-based & 11 & 140 & 518 & 658 & 124 & 556 & 680 \\
\hline Song 2008 & China & Asian & Hospital-based & 7 & 71 & 37 & 108 & 58 & 54 & 112 \\
\hline Altayli 2009 & Turkey & Caucasian & Hospital-based & 7 & 31 & 104 & 135 & 9 & 119 & 128 \\
\hline
\end{tabular}


Table 2 Characteristics of the studies evaluating effects of the GSTT1-null genotype of on bladder carcinogen risk (Continued)

\begin{tabular}{|c|c|c|c|c|c|c|c|c|c|c|}
\hline \multirow[t]{2}{*}{ Author, Year } & \multirow[t]{2}{*}{ Country } & \multirow[t]{2}{*}{ Ethnicity } & \multirow{2}{*}{$\begin{array}{l}\text { Source of } \\
\text { controls }\end{array}$} & \multirow{2}{*}{$\begin{array}{l}\text { Quality } \\
\text { score }\end{array}$} & \multicolumn{3}{|c|}{ Case } & \multicolumn{3}{|c|}{ Control } \\
\hline & & & & & - & + & total & - & + & total \\
\hline Grando 2009 & Brazil & Mix & Population-based & 7 & 51 & 49 & 100 & 37 & 63 & 100 \\
\hline Song 2009 & China & Asian & Hospital-based & 11 & 110 & 98 & 208 & 105 & 107 & 212 \\
\hline Cantor 2010 & Spain & Caucasian & Hospital-based & 9 & 136 & 542 & 678 & 160 & 550 & 710 \\
\hline Moore 2011 & USA & Mix & Hospital-based & 10 & 210 & 794 & 1004 & 237 & 942 & 1179 \\
\hline Rouissi 2011 & Tunisia & African & Population-based & 7 & 30 & 95 & 125 & 38 & 87 & 125 \\
\hline Goerlitz 2011 & Egypt & African & Hospital-based & 9 & 147 & 470 & 617 & 156 & 464 & 620 \\
\hline Salinas-Sánchez 2011 & Spain & Caucasian & Hospital-based & 5 & 42 & 148 & 190 & 25 & 138 & 163 \\
\hline Lesseur 2012 & New Hampshire & Caucasian & Hospital-based & 9 & 106 & 556 & 662 & 143 & 780 & 923 \\
\hline Ovsiannikov 2012 & Germany & Caucasian & Hospital-based & 6 & 33 & 163 & 196 & 47 & 188 & 235 \\
\hline $\begin{array}{l}\text { Henriquez- } \\
\text { Hernondez } 2012\end{array}$ & Spain & Caucasian & Hospital-based & 8 & 60 & 30 & 90 & 40 & 41 & 81 \\
\hline Berber 2013 & Turkey & Caucasian & Hospital-based & 7 & 31 & 83 & 114 & 16 & 98 & 114 \\
\hline Matic 2013 & Serbia & Caucasian & Hospital-based & 8 & 56 & 145 & 201 & 34 & 88 & 122 \\
\hline Safarinejad 2013 & Iran & Asian & Hospital-based & 10 & 35 & 131 & 166 & 69 & 263 & 332 \\
\hline Kang 2013 & Korea & Asian & Hospital-based & 9 & 64 & 46 & 110 & 128 & 92 & 220 \\
\hline Reszka 2014 & Poland & Caucasian & Population-based & 9 & 30 & 212 & 242 & 77 & 288 & 365 \\
\hline Ceylan 2015 & Turkey & Caucasian & Hospital-based & 8 & 19 & 46 & 65 & 9 & 61 & 70 \\
\hline Ali 2017 & Pakistan & Asian & Population-based & 11 & 34 & 166 & 200 & 26 & 174 & 200 \\
\hline Elhawary 2017 & Saudi Arabia & Asian & Hospital-based & 7 & 6 & 46 & 52 & 8 & 96 & 104 \\
\hline
\end{tabular}

Table 3 Characteristics of the studies evaluating effects of the GSTM1-GSTT1 dual-null genotype on bladder carcinogen risk

\begin{tabular}{|c|c|c|c|c|c|c|c|c|c|c|}
\hline \multirow[t]{2}{*}{ Author, Year } & \multirow[t]{2}{*}{ Country } & \multirow[t]{2}{*}{ Ethnicity } & \multirow{2}{*}{$\begin{array}{l}\text { Source of } \\
\text { controls }\end{array}$} & \multirow{2}{*}{$\begin{array}{l}\text { Quality } \\
\text { score }\end{array}$} & \multicolumn{3}{|l|}{ Case } & \multicolumn{3}{|l|}{ Control } \\
\hline & & & & & null-null & non-null-null & total & null-null & non-null-null & total \\
\hline Abdel-Rahman 1998 & Egypt & African & Hospital-based & 8 & 14 & 23 & 37 & 3 & 31 & 34 \\
\hline Steinhoff 2000 & Germany & Caucasian & Hospital-based & 7 & 12 & 123 & 135 & 4 & 123 & 127 \\
\hline Schnakenberg 2000 & Germany & Caucasian & Population-based & 6 & 12 & 145 & 157 & 31 & 192 & 223 \\
\hline Ma 2002 & China & Asian & Population-based & 8 & 16 & 45 & 61 & 54 & 128 & 182 \\
\hline Lee 2002 & Korea & Asian & Hospital-based & 8 & 83 & 149 & 232 & 37 & 128 & 165 \\
\hline Srivastava 2004 & India & Asian & Hospital-based & 7 & 16 & 90 & 106 & 9 & 173 & 182 \\
\hline Moore 2004 & USA & Mix & Population-based & 8 & 9 & 97 & 106 & 6 & 103 & 109 \\
\hline Hung 2004 & France & Caucasian & Hospital-based & 7 & 28 & 173 & 201 & 19 & 195 & 214 \\
\hline Srivastava 2005 & India & Asian & Population-based & 10 & 17 & 89 & 106 & 32 & 338 & 370 \\
\hline McGrath 2006 & USA & Mix & Population-based & 11 & 18 & 173 & 191 & 78 & 844 & 922 \\
\hline Song 2009 & China & Asian & Hospital-based & 11 & 77 & 131 & 208 & 50 & 162 & 212 \\
\hline Salinas-Sonchez 2011 & Spain & Caucasian & Hospital-based & 5 & 20 & 131 & 151 & 6 & 88 & 94 \\
\hline Ovsiannikov 2012 & Germany & Caucasian & Hospital-based & 6 & 17 & 179 & 196 & 29 & 206 & 235 \\
\hline Henriquez-Hernondez 2012 & Spain & Caucasian & Hospital-based & 8 & 17 & 73 & 90 & 8 & 73 & 81 \\
\hline Berber 2013 & Turkey & Caucasian & Hospital-based & 7 & 11 & 103 & 114 & 7 & 107 & 114 \\
\hline Safarinejad 2013 & Iran & Asian & Hospital-based & 10 & 38 & 128 & 166 & 73 & 259 & 332 \\
\hline Ceylan 2015 & Turkey & Caucasian & Hospital-based & 8 & 8 & 57 & 65 & 8 & 62 & 70 \\
\hline Elhawary 2017 & Saudi Arabia & Asian & Hospital-based & 7 & 0 & 104 & 104 & 0 & 208 & 208 \\
\hline
\end{tabular}




\begin{tabular}{|c|c|c|c|c|c|c|c|c|c|c|}
\hline & Studv or Subgroup & $\begin{array}{c}\text { Case } \\
\text { Events }\end{array}$ & Total & $\begin{array}{l}\text { Contr } \\
\text { Events }\end{array}$ & ${ }_{\text {Total }}$ & Weight & $\begin{array}{c}\text { Odds Ratio } \\
\text { M-H, Random, } 95 \% \mathrm{Cl}\end{array}$ & Year & $\begin{array}{r}\text { Odds } \\
M-H \text {, Rand }\end{array}$ & $\begin{array}{l}\text { Ratio } \\
\text { lom, } 95 \% \mathrm{Cl}\end{array}$ \\
\hline & Zhong 1993 & 39 & 97 & 94 & 225 & $1.1 \%$ & $0.94[0.58,1.52]$ & 1993 & & t \\
\hline & Bell 1993 & 111 & 200 & 85 & 200 & $1.4 \%$ & $1.69[1.14,2.51]$ & 1993 & & - \\
\hline & Daly 1993 & 45 & 53 & 31 & 58 & $0.4 \%$ & $4.90[1.97,12.20]$ & 1993 & & - \\
\hline & $\operatorname{Lin} 1994$ & 61 & 107 & 442 & 915 & $1.4 \%$ & $1.42[0.95,2.13]$ & 1994 & & - \\
\hline & Okkels 1996 & 133 & 233 & 100 & 200 & $1.5 \%$ & $1.33[0.91,1.94]$ & 1996 & & t- \\
\hline & Anwar 1996 & 19 & 22 & 10 & 21 & $0.2 \%$ & $6.97[1.57,30.87]$ & 1996 & & \\
\hline & Lafuente 1996 & 39 & 66 & 28 & 55 & $0.6 \%$ & $1.39[0.68,2.87]$ & 1996 & & - \\
\hline & Brockmaller 1996 & 217 & 374 & 192 & 373 & $2.0 \%$ & $1.30[0.98,1.74]$ & 1996 & & F- \\
\hline & Abdel-Rahman 1998 & 26 & 37 & 15 & 34 & $0.4 \%$ & $2.99[1.13,7.96]$ & 1998 & & $\longrightarrow$ \\
\hline & Katoh 1998 & 66 & 112 & 50 & 112 & $1.0 \%$ & $1.78[1.05,3.02]$ & 1998 & & - \\
\hline & Salagovic 1999 & 40 & 76 & 123 & 248 & $1.0 \%$ & $1.13[0.68,1.89]$ & 1999 & & $t$ \\
\hline & Schnakenberg 2000 & 93 & 157 & 129 & 223 & $1.4 \%$ & $1.06[0.70,1.60]$ & 2000 & & $t$ \\
\hline & Peluso 2000 & 61 & 130 & 29 & 54 & $0.7 \%$ & $0.76[0.40,1.44]$ & 2000 & & \\
\hline & Mungan 2000 & 38 & 61 & 30 & $\begin{array}{l}34 \\
69\end{array}$ & $0.6 \%$ & $2.15[1.06,4.34]$ & 2000 & & $\leftarrow$ \\
\hline & Kim 2000 & 78 & 112 & 128 & 225 & $1.1 \%$ & $1.74[1.07,2.81]$ & 2000 & & - \\
\hline & Georgiou 2000 & 56 & 89 & 56 & 147 & $0.9 \%$ & $2.76[1.60,4.75]$ & 2000 & & - \\
\hline & Steinhoff 2000 & 80 & 135 & 57 & 127 & $1.1 \%$ & $1.79[1.09,2.92]$ & 2000 & & - \\
\hline & Altas 2001 & 56 & 103 & 70 & 202 & $1.1 \%$ & $2.25[1.38,3.65]$ & 2001 & & - \\
\hline & Toruner 2001 & 75 & 121 & 55 & 121 & $1.0 \%$ & $1.96[1.17,3.27]$ & 2001 & & - \\
\hline & Lee 2002 & 149 & 232 & 86 & 165 & $1.4 \%$ & $1.65[1.10,2.48]$ & 2002 & & - \\
\hline & Kim 2002 & 138 & 216 & 265 & 449 & $1.7 \%$ & $1.23[0.88,1.72]$ & 2002 & & F \\
\hline & Giannakopoulos 2002 & 56 & 89 & 56 & 147 & $0.9 \%$ & $2.76[1.60,4.75]$ & 2002 & & - \\
\hline & Ma 2002 & 180 & 317 & 99 & 182 & $1.6 \%$ & $1.10[0.76,1.59]$ & 2002 & & t \\
\hline & Schroeder 2003 & 137 & 230 & 101 & 213 & $1.5 \%$ & $1.63[1.12,2.38]$ & 2003 & & - \\
\hline & Jong 2003 & 75 & 126 & 99 & 204 & $1.2 \%$ & $1.56[1.00,2.44]$ & 2003 & & - \\
\hline & Moore 2004 & 54 & 106 & 49 & 109 & $1.0 \%$ & $1.27[0.74,2.17]$ & 2004 & & $\leftarrow$ \\
\hline & Srivastawa 2004 & 42 & 106 & 54 & 182 & $1.0 \%$ & $1.56[0.94,2.57]$ & 2004 & & - \\
\hline & Hung 2004 & 132 & 201 & 112 & 214 & $1.4 \%$ & $1.74[1.17,2.59]$ & 2004 & & - \\
\hline & Saad 2005 & 45 & 72 & 40 & 81 & $0.7 \%$ & $1.71[0.90,3.26]$ & 2005 & & - \\
\hline & Srivastava 2005 & 140 & 370 & 43 & 106 & $1.3 \%$ & $0.89[0.57,1.39]$ & 2005 & & - \\
\hline & Sobti 2005 & 37 & 100 & 24 & 76 & $0.7 \%$ & $1.27[0.68,2.39]$ & 2005 & & - \\
\hline & Garcia-Closas 2005 & 716 & 1138 & 571 & 1132 & $2.8 \%$ & $1.67[1.41,1.97]$ & 2005 & & - \\
\hline & Karagas 2005 & 210 & 344 & 309 & 542 & $2.1 \%$ & $1.18[0.90,1.56]$ & 2005 & & t- \\
\hline & $\operatorname{Kim} 2005$ & 92 & 153 & 73 & 153 & $1.2 \%$ & $1.65[1.05,2.60]$ & 2005 & & - \\
\hline & McGrath 2006 & 109 & 191 & 483 & 922 & $1.8 \%$ & $1.21[0.88,1.65]$ & 2006 & & t \\
\hline & Ouerhani 2006 & 39 & 62 & 36 & 79 & $0.7 \%$ & $2.03[1.03,4.00]$ & 2006 & & - \\
\hline & Murta-Nascimento 2007 & 428 & 679 & 367 & 735 & $2.5 \%$ & $1.71[1.38,2.11]$ & 2007 & & - \\
\hline & Moore 2007 & 683 & 1077 & 524 & 1022 & $2.7 \%$ & $1.65[1.38,1.96]$ & 2007 & & - \\
\hline & Kellen 2007 & 312 & 579 & 597 & 1063 & $2.5 \%$ & $0.91[0.74,1.12]$ & 2007 & & $t$ \\
\hline & Cengiz 2007 & 34 & 51 & 22 & 53 & $0.5 \%$ & $2.82[1.27,6.26]$ & 2007 & & 一 \\
\hline & Zhao 2007 & 324 & 622 & 317 & 633 & $2.4 \%$ & $1.08[0.87,1.35]$ & 2007 & & $t$ \\
\hline & Shao 2008 & 85 & 202 & 81 & 272 & $1.5 \%$ & $1.71[1.17,2.51]$ & 2008 & & - \\
\hline & Covolo 2008 & 128 & 197 & 111 & 211 & $1.4 \%$ & $1.67[1.12,2.49]$ & 2008 & & - \\
\hline & Yuan 2008 & 387 & 662 & 335 & 686 & $2.5 \%$ & $1.47[1.19,1.83]$ & 2008 & & - \\
\hline & Golka 2008 & 184 & 293 & 88 & 176 & $1.5 \%$ & $1.69[1.16,2.47]$ & 2008 & & - \\
\hline & Song 2009 & 131 & 208 & 108 & 212 & $1.5 \%$ & $1.64[1.11,2.42]$ & 2009 & & - \\
\hline & Grando 2009 & 40 & 100 & 33 & 100 & $0.9 \%$ & $1.35[0.76,2.41]$ & 2009 & & $\leftarrow$ \\
\hline & Altayli 2009 & 58 & 135 & 65 & 128 & $1.1 \%$ & $0.73[0.45,1.19]$ & 2009 & & $t$ \\
\hline & Zupa 2009 & 13 & 23 & 68 & 121 & $0.4 \%$ & $1.01[0.41,2.49]$ & 2009 & & E \\
\hline & $\operatorname{Lin} 2009$ & 312 & 604 & 286 & 610 & $2.4 \%$ & $1.21[0.97,1.52]$ & 2009 & & - \\
\hline & Abd 2010 & 11 & 20 & 9 & 20 & $0.2 \%$ & $1.49[0.43,5.19]$ & 2010 & & 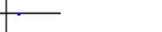 \\
\hline & Salinas-Sonchez 2011 & 109 & 201 & 78 & 193 & $1.4 \%$ & $1.75[1.17,2.60]$ & 2011 & & - \\
\hline & Goerlite 2011 & 344 & 618 & 332 & 621 & $2.4 \%$ & $1.09[0.87,1.37]$ & 2011 & & $t$ \\
\hline & Moore 2011 & 653 & 1053 & 690 & 1235 & $2.8 \%$ & $1.29[1.09,1.52]$ & 2011 & & - \\
\hline & Rouissi 2011 & 63 & 125 & 56 & 125 & $1.1 \%$ & $1.25[0.76,2.06]$ & 2011 & & - \\
\hline & Öztürk 2011 & 98 & 176 & 51 & 97 & $1.1 \%$ & $1.13[0.69,1.86]$ & 2011 & & t- \\
\hline & Marenne 2012 & 488 & 773 & 402 & 759 & $2.5 \%$ & $1.52[1.24,1.86]$ & 2012 & & - \\
\hline & Ovsiannikov 2012 & 102 & 196 & 123 & 235 & $1.5 \%$ & $0.99[0.68,1.44]$ & 2012 & & $t$ \\
\hline & Lesseur 2012 & 378 & 653 & 508 & 928 & $2.5 \%$ & $1.14[0.93,1.39]$ & 2012 & & $t$ \\
\hline & Zhang 2012 & 381 & 710 & 402 & 782 & $2.5 \%$ & $1.09[0.89,1.34]$ & 2012 & & $t$ \\
\hline & Schwender 2012 & 909 & 1572 & 863 & 1739 & $3.0 \%$ & $1.39[1.21,1.60]$ & 2012 & & - \\
\hline & Henríquez-Hernández 2012 & 23 & 90 & 17 & 81 & $0.6 \%$ & $1.29[0.63,2.64]$ & 2012 & & - \\
\hline & Safarinejad 2013 & 50 & 166 & 93 & 332 & $1.4 \%$ & $1.11[0.74,1.67]$ & 2013 & & - \\
\hline & Wang 2013 & 699 & 1050 & 834 & 1404 & $2.8 \%$ & $1.36[1.15,1.61]$ & 2013 & & - \\
\hline & Matic 2013 & 111 & 201 & 61 & 122 & $1.2 \%$ & $1.23[0.79,1.94]$ & 2013 & & $\leftarrow$ \\
\hline & Savic-Radojevic 2013 & 45 & 80 & 32 & 60 & $0.7 \%$ & $1.13[0.57,2.20]$ & 2013 & & t \\
\hline & Berber 2013 & 54 & 114 & 51 & 114 & $1.0 \%$ & $1.11[0.66,1.87]$ & 2013 & & t \\
\hline & Kang 2013 & 65 & 110 & 103 & 220 & $1.2 \%$ & $1.64[1.03,2.61]$ & 2013 & & - \\
\hline & Reszka 2014 & 149 & 244 & 165 & 365 & $1.7 \%$ & $1.90[1.37,2.64]$ & 2014 & & - \\
\hline & Ceylan 2015 & 22 & 65 & 31 & 70 & $0.6 \%$ & $0.64[0.32,1.29]$ & 2015 & & $t$ \\
\hline & Ali 2017 & 83 & 200 & 57 & 200 & $1.3 \%$ & $1.78[1.17,2.70]$ & 2017 & & - \\
\hline & Elhawary 2017 & 24 & 52 & 40 & 104 & $0.7 \%$ & $1.37[0.70,2.69]$ & 2017 & & - \\
\hline & & & 20239 & & 24393 & $100.0 \%$ & $1.40[1.31,1.48]$ & & & 1 \\
\hline & Total events & 11664 & (a) & 12224 & (n) & & & & & \\
\hline & $\begin{array}{l}\text { Heterogeneity: Tau }=0.03 ; \mathrm{Cl} \\
\text { Test for overall effect: } Z=10.6\end{array}$ & $\begin{array}{l}h^{2}=140.13 \\
3\langle(P=0.00\end{array}$ & $\begin{array}{l}3, \mathrm{df}=71 \\
0001)\end{array}$ & $1(P<0.0$ & $00001) ; 1^{2}$ & $F^{2}=49 \%$ & & & $\begin{array}{|cc|}0.01 & 0.1 \\
& \text { Favours case }\end{array}$ & 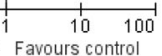 \\
\hline
\end{tabular}

cancer risk in the overall population, Asians and controls from both hospital-based and population-based studies that included both high- and low-quality studies. However, we did not find this relationship in whites or Africans (Table 4).

\section{Association of the GSTT1-null genotype with bladder cancer risk}

In this study, we found that the GSTT1-null genotype was associated with bladder cancer risk in the overall population, and controls from hospital-based studies 
Table 4 Meta-analysis of the association of null genotypes of GSTM1, GST11 and dual-null genotype of GSTM1/GST1 with bladder carcinogens risk

\begin{tabular}{|c|c|c|c|c|c|c|}
\hline Genetic contrasts & Group and subgroups & Studies Number & $Q$ test $P$ value & Model selected & OR $(95 \% \mathrm{Cl})$ & $P$ \\
\hline \multicolumn{7}{|l|}{ GSTM1 } \\
\hline \multirow[t]{8}{*}{$-v s+$} & Overall & 72 & $<0.00001$ & Random & $1.40(1.31,1.48)$ & $<0.00001$ \\
\hline & Caucasian & 37 & $<0.00001$ & Random & $1.39(1.26,1.54)$ & $<0.00001$ \\
\hline & Asian & 20 & 0.39 & Fixed & $1.45(1.33,1.59)$ & $<0.00001$ \\
\hline & African & 9 & 0.10 & Random & $1.54(1.16,2.05)$ & 0.003 \\
\hline & Hospital-based & 46 & 0.0001 & Random & $1.42(1.32,1.52)$ & $<0.00001$ \\
\hline & Population-based & 26 & 0.003 & Random & $1.36(1.21,1.53)$ & $<0.00001$ \\
\hline & High quality & 54 & 0.0002 & Random & $1.37(1.28,1.45)$ & $<0.00001$ \\
\hline & Low quality & 18 & 0.0009 & Random & $1.58(1.29,1.94)$ & $<0.0001$ \\
\hline \multicolumn{7}{|l|}{ GSTM1 (smoking) } \\
\hline \multirow[t]{6}{*}{$-v s+$} & Overall & 24 & 0.02 & Random & $1.37(1.19,1.59)$ & $<0.0001$ \\
\hline & Caucasian & 10 & 0.007 & Random & $1.17(0.85,1.59)$ & 0.33 \\
\hline & Asian & 7 & 0.63 & Fixed & $1.67(1.32,2.11)$ & $<0.0001$ \\
\hline & African & 3 & 0.22 & Fixed & $1.44(0.95,2.17)$ & 0.08 \\
\hline & High quality & 17 & 0.02 & Random & $1.35(1.14,1.60)$ & 0.0005 \\
\hline & Low quality & 7 & 0.27 & Fixed & $1.48(1.12,1.96)$ & 0.006 \\
\hline \multicolumn{7}{|l|}{ GSTT1 } \\
\hline \multirow[t]{8}{*}{$-v s+$} & Overall & 61 & $<0.00001$ & Random & $1.11(1.01,1.22)$ & 0.03 \\
\hline & Caucasian & 29 & $<0.00001$ & Random & $1.16(0.99,1.36)$ & 0.07 \\
\hline & Asian & 21 & 0.01 & Random & $1.05(0.91,1.22)$ & 0.51 \\
\hline & African & 4 & 0.03 & Random & $1.07(0.65,1.76)$ & 0.79 \\
\hline & Hospital-based & 40 & $<0.0001$ & Random & $1.11(0.99,1.24)$ & 0.07 \\
\hline & Population-based & 21 & 0.0002 & Random & $1.12(0.94,1.35)$ & 0.20 \\
\hline & High quality & 52 & $<0.00001$ & Random & $1.14(1.03,1.26)$ & 0.01 \\
\hline & Low quality & 9 & 0.23 & Fixed & $0.93(0.75,1.14)$ & 0.49 \\
\hline \multicolumn{7}{|l|}{ GSTT1 (smoking) } \\
\hline & Overall & 21 & 0.67 & Fixed & $1.06(0.93,1.20)$ & 0.38 \\
\hline & Caucasian & 9 & 0.84 & Fixed & $1.14(0.91,1.43)$ & 0.24 \\
\hline & Asian & 7 & 0.62 & Fixed & $1.00(0.77,1.30)$ & 0.99 \\
\hline & African & 2 & 0.41 & Fixed & $0.60(0.36,1.02)$ & 0.06 \\
\hline & High quality & 16 & 0.52 & Fixed & $1.06(0.93,1.22)$ & 0.37 \\
\hline & Low quality & 5 & 0.64 & Fixed & $1.01(0.70,1.48)$ & 0.94 \\
\hline \multicolumn{7}{|c|}{ Dual-null genotype of GSTM1/GSTT1 } \\
\hline & Overall & 18 & 0.003 & Random & $1.48(1.15,1.92)$ & 0.002 \\
\hline & Caucasian & 8 & 0.03 & Random & $1.30(0.83,2.03)$ & 0.25 \\
\hline & Asian & 7 & 0.04 & Random & $1.62(1.15,2.28)$ & 0.006 \\
\hline & Hospital-based & 13 & 0.03 & Random & $1.71(1.28,2.28)$ & 0.0003 \\
\hline & Population-based & 5 & 0.06 & Random & $1.07(0.67,1.71)$ & 0.77 \\
\hline & High quality & 15 & 0.11 & Fixed & $1.61(1.36,1.91)$ & $<0.00001$ \\
\hline & Low quality & 3 & 0.04 & Random & $0.86(0.40,1.85)$ & 0.70 \\
\hline
\end{tabular}

that included high-quality studies; but not with bladder cancer risk in whites, Africans, Asians or controls from population-based studies that included low-quality studies (overall population: $\mathrm{OR}=1.11,95 \%$ CI: $1.01-1.22, P=0.03$; whites: $\mathrm{OR}=1.16,95 \% \mathrm{CI}$ : $0.99-1.36, P=0.07$; Africans: $\mathrm{OR}=1.07,95 \% \mathrm{CI}: 0.65-$ 
1.76, $P=0.79$; Asians: $\mathrm{OR}=1.05,95 \% \mathrm{CI}: 0.91-1.22$, $P=0.51$; Fig. 3 for overall population; Table 4). However, in controls from either hospital-based or population-based studies that included both high- and low-quality studies, or in the meta-analysis for all patients and controls who smoked cigarettes, we found that the GSTT1-null genotype was not associated with bladder cancer risk in the overall population, or in individual white, African or Asian populations (Table 4).

\section{Association of dual-null GSTM1-GSTT1 genotype with bladder cancer risk}

We found an association between the dual-null GSTM1-GSTT1 genotype and bladder cancer risk in the overall population, Asians and controls from hospital-based studies that included high-quality studies (overall population: $\mathrm{OR}=1.48,95 \% \mathrm{CI}: 1.15-1.92, P=$ 0.002; Asians: $\mathrm{OR}=1.62,95 \% \mathrm{CI}: 1.15-2.28, P=0.006$; Fig. 4 for overall population; Table 4). However, the dual-null GSTM1-GSTT1 genotype was not associated

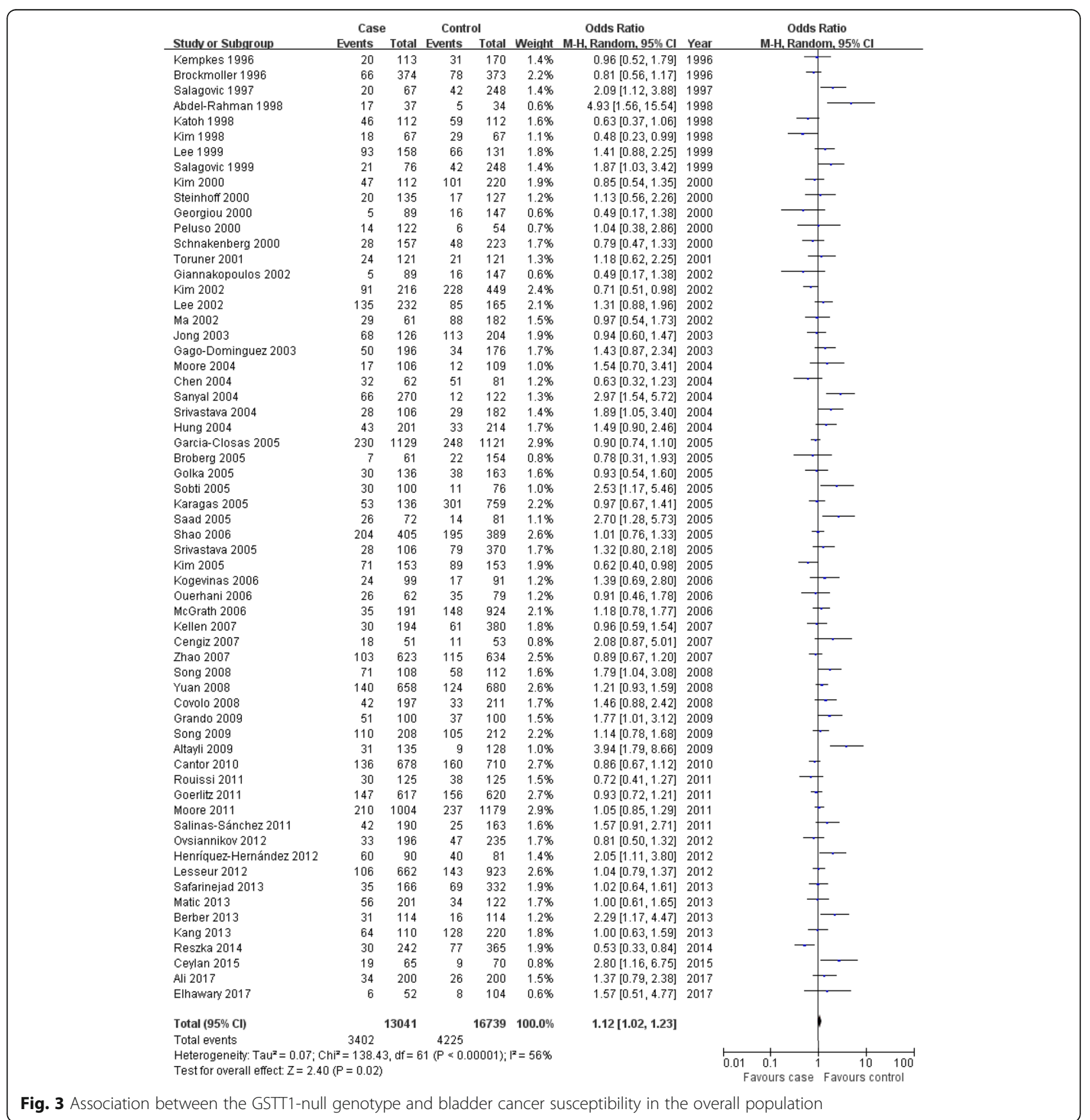




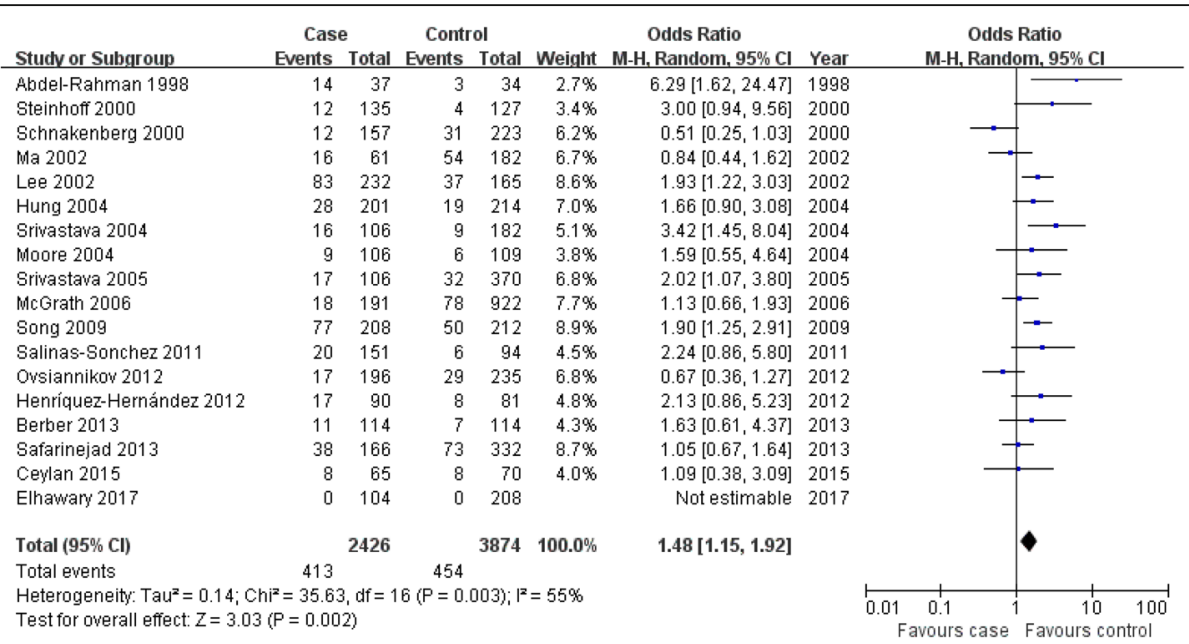

Fig. 4 Association between the dual-null GSTM1-GSTT1 genotype and bladder cancer risk in the overall population

with onset of bladder cancer in whites or in controls from population-based studies that included low-quality studies (whites: $\mathrm{OR}=1.30,95 \% \mathrm{CI}: 0.83-2.03, P=$ 0.25 ; Table 4).

\section{Evaluation of publication bias}

We performed a publication bias test for the association of the GSTM1-null, GSTT1-null and dual-null GSTM1-GSTT1 genotypes with bladder cancer risk in the overall population. There was no bias for the association of the dual-null GSTM1-GSTT1 genotype with bladder cancer risk, but there was for the GSTM1- and
GSTT1-null genotypes (GSTM1-null: Begg $P=0.100$, Egger $P=0.052$; GSTT1-null: Begg $P=0.001$, Egger $P=$ 0.002; dual-null GSTM1-GSTT1: Begg $P=0.343$, Egger $P=0.236$; Fig. 5).

\section{Discussion}

Research on single-nucleotide polymorphisms have focused mainly on their impact on tumor suppressor genes, metabolic-enzyme genes, and DNA repair genes, etc. Understanding disease susceptibility and pathogenesis and using them to guide diagnosis and individual treatment choice constitute an important
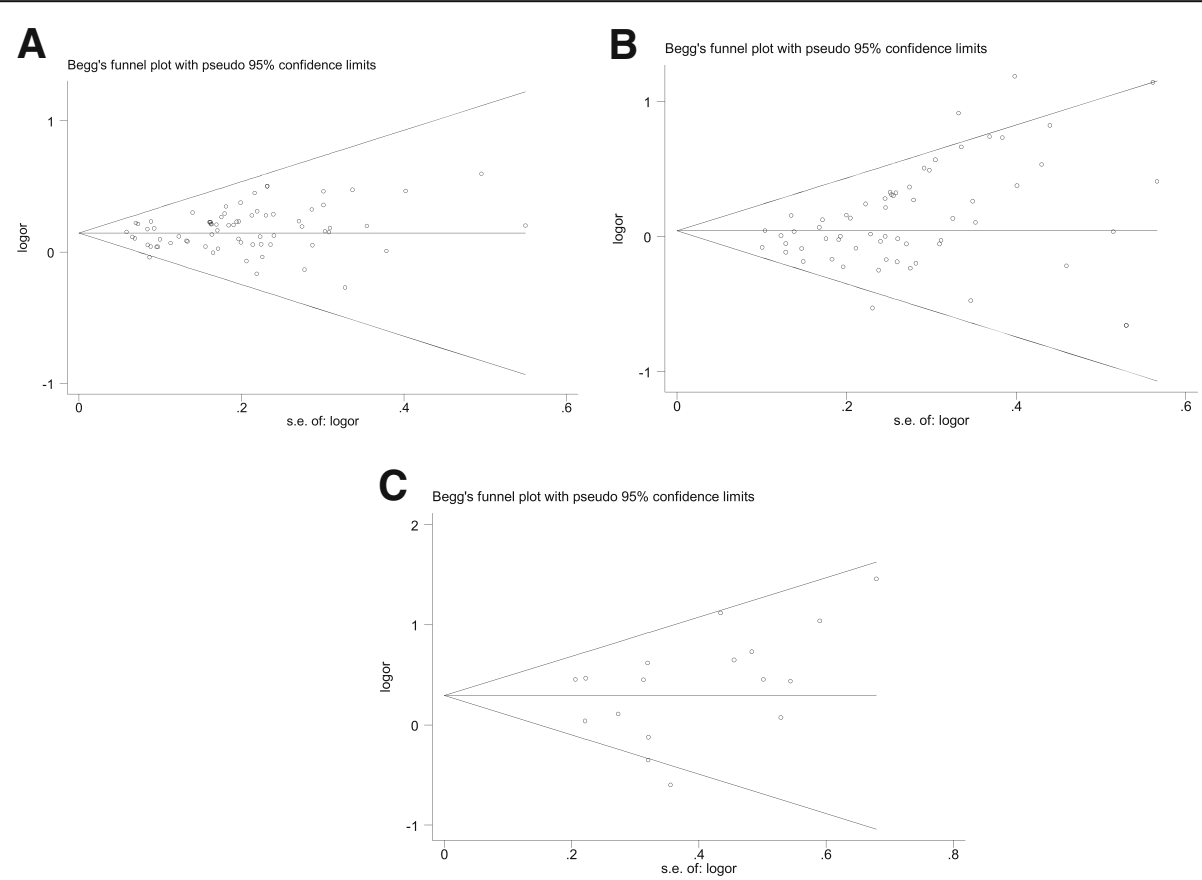

Fig. 5 Publication bias. a GSTM1-null genotype. b GSTT1-null genotype. c Dual-null GSTM1-GSTT1 genotype 
new therapeutic approach [109]. In this study, we found that the average distribution frequency of the GSTM1-null genotype was significantly higher in bladder cancer cases than in controls (case/control =1.20). In the subgroup of patients and controls who smoked cigarettes, it was also higher in the bladder cancer case group compared with the control group (case/control=1.17). This might indicate that the GSTM1-null genotype was associated with bladder cancer risk in the overall population, including whites, Africans, Asians, and controls from both hospital-based and population-based studies that included both high- and low-quality studies. In the meta-analysis for all patients and controls who smoked cigarettes, we found that the GSTM1-null genotype was associated with bladder cancer risk in the overall population, Asians, and controls from both hospital-based and population-based studies that included both high- and low-quality studies. The sample size of our meta-analysis was larger than those of other meta-analyses [61, 110-112], and therefore our results might be more robust. However, our tests for publication bias, the GSTM1 studies were found to be positive. Therefore, the positive association between the GSTM1-null genotype and bladder cancer should be reassessed in the future.

The average distribution frequency of the GSTT1-null genotype was higher in the bladder cancer case group than in the control group (case/control $=1.11$ ). In the subgroup of patients and controls who smoked cigarettes, it was similar in both groups (case/control $=1.02$ ). This might tell us that the GSTT1-null genotype was associated with bladder cancer risk. For confirmation, we performed a meta-analysis, which further showed the GSTT1-null genotype to be associated with bladder cancer risk in the overall population, whites and controls from hospital-based studies that included high-quality studies. In the meta-analysis for all patients and controls who smoked cigarettes, we found that the GSTT1-null genotype was not associated with bladder cancer risk in the overall population, whites, Africans, Asians or controls from both hospital-based and population-based studies that included both high- and low-quality studies. Our results indicate that the GSTT1-null genotype does not predict the risk of bladder cancer. The sample size of our meta-analysis was larger than those of other meta-analyses [111, 112], suggesting that our conclusion might be more robust. However, publication bias was also found for GSTT1. Therefore, further studies are required.

Average distribution frequency of the dual-null GSTM1-GSTT1 genotype in the bladder cancer group was slightly higher than in the control group (case/control $=1.47$ ), indicating a possible association between the dual-null GSTM1-GSTT1 genotype and bladder cancer risk. Meta-analysis further revealed an association between the dual-null GSTM1-GSTT1 genotype and bladder cancer risk in the overall population, Asians and controls from hospital-based studies that included high-quality studies. No publication bias was found for this meta-analysis, and the conclusion was robust.

In a previous study, García-Closas et al. [61] conducted a meta-analysis of 28 studies of GSTM1 and reported that the GSTM1-null genotype both increased the overall risk of bladder cancer and posed similar relative risks for both smokers and non-smokers. This finding suggested that GSTM1 lowers the risk of bladder cancer through mechanisms that are not specific to the detoxification of polycyclic aromatic hydrocarbons in tobacco smoke. Engel et al. [110] performed a meta-analysis of GSTM1 and bladder cancer that included 17 studies and reported that the GSTM1-null status is associated with a modest increase in the risk of bladder cancer, and that there was no evidence of multiplicative interaction between the GSTM1-null genotype and once and current smoking in relation to bladder cancer. A meta-analysis by $\mathrm{Yu}$ et al. [112] included 48 case-control studies for GSTM1-null and 57 studies for GSTT1, and suggested that the GSTM1- and GSTT1-null genotypes might both be related to higher bladder cancer risk. Yu et al. [111] also performed a meta-analysis to investigate the association between GSTM1-GSTT1 deletion polymorphisms and bladder cancer susceptibility, including 46 studies of GSTM1-null, 54 of GSTT1 and 10 of dual-null GSTM1-GSTT1. All 3 genotypes were associated with increased bladder cancer risk. In our meta-analysis, we included 72 studies for GSTM1-null, 62 for GSTT1-null and 18 for dual-null GSTM1-GSTT1 genotypes. These results from the meta-analyses mentioned above were similar to our results. However, the sample size of our meta-analysis was larger than the previous meta-analyses, and the results from our studies might be more robust. Furthermore, we initially conducted a meta-analysis that showed no evidence of multiplicative interaction between the GSTT1-null genotype and smoking in relation to bladder cancer.

Smoking is a known risk factor for bladder cancer [113], and the products of GSTs help detoxify the polycyclic aromatic hydrocarbons found in tobacco smoke [114]. Our study suggests that the GSTM1-null genotype might play a role in such detoxification, but the GSTT1-null genotype does not. However, more studies should be conducted to confirm this.

GSTM1-null, GSTT1-null and dual-null GSTM1-GSTT1 genotypes play an important role in detoxification of various toxic compounds, such as carcinogens. In this meta-analysis, it indicated that GSTM1-null, GSTT1-null and dual-null GSTM1-GSTT1 genotypes were risk factors to susceptibility of bladder cancer, and took part in the pathogenesis of bladder cancer. 
There were limitations in our meta-analysis. First, age might be a source of heterogeneity, but it was difficult to stratify the different ages in the reports prior to pooling the results, for the reason that the ages from most of the included studies were different. So, no conclusions can be drawn regarding the impact of GSTs on age of onset. Furthermore, heterogeneity and publication bias were both significant for GSTM1-null and GSTT1-null. Subgroup analyses were performed to find out any effect modifier, but the reason was not clear.

\section{Conclusion}

Our results supported that the GSTM1-null, GSTT1-null and dual-null GSTM1-GSTT1 genotypes might be associated with the onset of bladder cancers. However, more association investigations are required to further clarify these relationships.

\section{Additional file}

Additional file 1: Table S1. Scale for Quality Assessment. (DOC 42 kb)

\section{Acknowledgements \\ Not applicable.}

\section{Funding}

This study was supported by Guangzhou Medical Key Discipline Construction Project. The funding paid the publication fee for this paper.

\section{Availability of data and materials}

The datasets used and/or analyzed during the current study are available from the corresponding author on reasonable request.

\section{Authors' contributions}

TBZ was in charge of conceived and designed the study. TBZ, HYL, WJX, ZQZ, HZZ were responsible for collection of data and performing the statistical analysis and manuscript preparation. TBZ and ZJL were responsible for checking the data. All authors were responsible for drafting the manuscript, read and approved the final version.

\section{Ethics approval and consent to participate}

Not applicable.

\section{Consent for publication}

Not applicable.

\section{Competing interests}

The authors declare that they have no competing interests.

\section{Publisher's Note}

Springer Nature remains neutral with regard to jurisdictional claims in published maps and institutional affiliations.

\section{Author details}

${ }^{1}$ Department of Nephrology, The Second Affiliated Hospital of Shantou University Medical College, Shantou 515041, China. ${ }^{2}$ Department of Nephrology, Huadu District People's Hospital of Guangzhou, Southern Medical University, Guangzhou, China.
Received: 16 November 2017 Accepted: 30 October 2018

Published online: 12 November 2018

\section{References}

1. John BA, Said N. Insights from animal models of bladder cancer: recent advances, challenges, and opportunities. Oncotarget. 2017:8:57766-81.

2. Chen $\mathrm{CH}$, Shun $\mathrm{CT}$, Huang $\mathrm{KH}$, Huang CY, Yu HJ, Pu YS. Characteristics of female non-muscle-invasive bladder cancer in Taiwan: association with upper tract urothelial carcinoma and end-stage renal disease. Urology. 2008; 71:1155-60

3. Zhang Z, Zhang G, Gao Z, Li S, Li Z, Bi J, Liu X, Kong C. Comprehensive analysis of differentially expressed genes associated with PLK1 in bladder cancer. BMC Cancer. 2017;17:861.

4. Oliveira PA, Vasconcelos-Nobrega C, Gil da Costa RM, Arantes-Rodrigues R The N-butyl-N-4-hydroxybutyl nitrosamine mouse urinary bladder Cancer model. Methods Mol Biol. 2018;1655:155-67.

5. Reszka E. Selenoproteins in bladder cancer. Clin Chim Acta. 2012;413:84754.

6. Chen $\mathrm{CH}$, Chiou HY, Hsueh YM, Chen CJ, Yu HJ, Pu YS. Clinicopathological characteristics and survival outcome of arsenic related bladder cancer in Taiwan. J Urol. 2009;181:547-52 discussion 553.

7. Benhamou S, Bonastre J, Groussard K, Radvanyi F, Allory Y, Lebret T. A prospective multicenter study on bladder cancer: the COBLAnCE cohort. BMC Cancer. 2016:16:837.

8. Verma A, Kapoor R, Mittal RD. Cluster of differentiation 44 (CD44) gene variants: a putative Cancer stem cell marker in risk prediction of bladder Cancer in north Indian population. Indian J Clin Biochem. 2017;32:74-83.

9. Singh $V$, Jaiswal PK, Mittal RD. Replicative study of GWAS TP63C/T, TERTC/T, and SLC14A1C/T with susceptibility to bladder cancer in north Indians. Urol Oncol. 2014;32:1209-14.

10. Wieczorek E, Wasowicz W, Gromadzinska J, Reszka E. Functional polymorphisms in the matrix metalloproteinase genes and their association with bladder cancer risk and recurrence: a mini-review. Int J Urol. 2014;21:744-52.

11. Selinski S. Discovering urinary bladder cancer risk variants: status quo after almost ten years of genome-wide association studies. EXCLI J. 2017;16:1288-96.

12. Selinski S, Blaszkewicz M, Ickstadt $K$, Gerullis $H$, Otto T, Roth E, Volkert F, Ovsiannikov D, Moormann O, Banfi G, Nyirady P, Vermeulen SH, Garcia-Closas M, Figueroa JD, Johnson A, Karagas MR, Kogevinas M, Malats N, Schwenn M, Silverman DT, Koutros S, Rothman N, Kiemeney LA, Hengstler JG, Golka K. Identification and replication of the interplay of four genetic high-risk variants for urinary bladder cancer. Carcinogenesis. 2017;38:1167-79.

13. Mittal RD, Kesarwani $P$, Singh R, Ahirwar D, Mandhani A. GSTM1, GSTM3 and GSTT1 gene variants and risk of benign prostate hyperplasia in North India. Dis Markers. 2009;26:85-91.

14. Tharuka M, Bathige S, Lee J. Molecular cloning, biochemical characterization, and expression analysis of two glutathione S-transferase paralogs from the bigbelly seahorse (Hippocampus abdominalis). Comp Biochem Physiol B Biochem Mol Biol. 2017:4(214):1-11. https://doi.org/10.1016/j.cbpb.2017.08.005.

15. Mi Y, Ren K, Zou J, Bai Y, Zhang L, Zuo L, Okada A, Yasui T. The association between three genetic variants in MicroRNAs (Rs11614913, Rs2910164, Rs3746444) and prostate Cancer risk. Cell Physiol Biochem. 2018;48:149-57.

16. Baltruskeviciene E, Kazbariene B, Aleknavicius E, Krikstaponiene A, Venceviciene L, Suziedelis K, Stratilatovas E, Didziapetriene J. Changes of reduced glutathione and glutathione S-transferase levels in colorectal cancer patients undergoing treatment. Tumori 2017:0.

17. Muguruma N, Okamoto K, Nakagawa T, Sannomiya K, Fujimoto S, Mitsui $Y$, Kimura T, Miyamoto H, Higashijima J, Shimada M, Horino Y, Matsumoto S, Hanaoka K, Nagano T, Shibutani M, Takayama T. Molecular imaging of aberrant crypt foci in the human colon targeting glutathione S-transferase P1-1. Sci Rep 2017;7:6536.

18. Wang W, Liu F, Wang C, Tang Y, Jiang Z. Glutathione S-transferase A1 mediates nicotine-induced lung cancer cell metastasis by promoting epithelial-mesenchymal transition. Exp Ther Med. 2017;14:1783-8.

19. Rao A, Parameswar P, Majumdar S, Uppala D, Kotina S, Vennamaneni N. Evaluation of genetic polymorphisms in glutathione S-transferase Theta1, glutathione S-transferase Mu1, and glutathione S-transferase Mu3 in Oral leukoplakia and Oral squamous cell carcinoma with deleterious habits using polymerase chain reaction. Int J Appl Basic Med Res. 2017;7:181-5.

20. Zhao E, Hu K, Zhao Y. Associations of the glutathione S-transferase P1 lle105Val genetic polymorphism with gynecological cancer susceptibility: a meta-analysis. Oncotarget. 2017;8:41734-9. 
21. Xue C, He X, Zou D. Glutathione S-transferase M1 polymorphism and breast Cancer risk: a meta-analysis in the Chinese population. Clin Lab. 2016;62: 2277-84.

22. Thakkinstian A, McEvoy M, Minelli C, Gibson P, Hancox B, Duffy D, Thompson J, Hall I, Kaufman J, Leung TF, Helms PJ, Hakonarson H, Halpi E, Navon R, Attia J. Systematic review and meta-analysis of the association between \{beta\}2-adrenoceptor polymorphisms and asthma: a HuGE review. Am J Epidemiol. 2005;162:201-11.

23. Begg CB, Mazumdar M. Operating characteristics of a rank correlation test for publication bias. Biometrics. 1994;50:1088-101.

24. Egger M, Davey Smith G, Schneider M, Minder C. Bias in meta-analysis detected by a simple, graphical test. BMJ. 1997;315:629-34

25. Bell DA, Taylor JA, Paulson DF, Robertson CN, Mohler JL, Lucier GW. Genetic risk and carcinogen exposure: a common inherited defect of the carcinogen-metabolism gene glutathione S-transferase M1 (GSTM1) that increases susceptibility to bladder cancer. J Natl Cancer Inst. 1993;85:115964.

26. Zhong S, Wyllie AH, Barnes D, Wolf CR, Spurr NK. Relationship between the GSTM1 genetic polymorphism and susceptibility to bladder, breast and colon cancer. Carcinogenesis. 1993;14:1821-4.

27. Daly AK, Thomas DJ, Cooper J, Pearson WR, Neal DE, Idle JR. Homozygous deletion of gene for glutathione S-transferase $\mathrm{M} 1$ in bladder cancer. BMJ. 1993;307:481-2

28. Lin HJ, Han CY, Bernstein DA, Hsiao W, Lin BK, Hardy S. Ethnic distribution of the glutathione transferase mu 1-1 (GSTM1) null genotype in 1473 individuals and application to bladder cancer susceptibility. Carcinogenesis. 1994:15:1077-81.

29. Brockmoller J, Cascorbi I, Kerb R, Roots I. Combined analysis of inherited polymorphisms in arylamine $\mathrm{N}$-acetyltransferase 2, glutathione S-transferases M1 and T1, microsomal epoxide hydrolase, and cytochrome P450 enzymes as modulators of bladder cancer risk. Cancer Res. 1996;56:3915-25.

30. Lafuente A, Zakahary MM, El-Aziz MA, Ascaso C, Lafuente MJ, Trias M, Carretero P. Influence of smoking in the glutathione-S-transferase M1 deficiency--associated risk for squamous cell carcinoma of the bladder in schistosomiasis patients in Egypt. Br J Cancer. 1996;74:836-8.

31. Okkels $H$, Sigsgaard $T$, Wolf $H$, Autrup $H$. Glutathione S-transferase mu as a risk factor in bladder tumours. Pharmacogenetics. 1996;6:251-6.

32. Abdel-Rahman SZ, Anwar WA, Abdel-Aal WE, Mostafa HM, Au WW. GSTM1 and GSTT1 genes are potential risk modifiers for bladder cancer. Cancer Detect Prev. 1998;22:129-38.

33. Katoh $T$, Inatomi H, Kim H, Yang M, Matsumoto T, Kawamoto T. Effects of glutathione S-transferase (GST) M1 and GSTT1 genotypes on urothelial cancer risk. Cancer Lett. 1998;132:147-52.

34. Salagovic J, Kalina I, Habalova V, Hrivnak M, Valansky L, Biros E. The role of human glutathione S-transferases M1 and T1 in individual susceptibility to bladder cancer. Physiol Res. 1999;48:465-71.

35. Mungan NA, Aben KK, Beeks E, Kampman E, Bunschoten A, Bussemakers M, Witjes JA, Kiemeney LA. A germline homozygote deletion of the glutathione-S-transferase Mu1 gene predisposes to bladder cancer. Urol Int. 2000;64:134-8.

36. Georgiou I, Filiadis IF, Alamanos Y, Bouba I, Giannakopoulos X, Lolis D. Glutathione S-transferase null genotypes in transitional cell bladder cancer: a case-control study. Eur Urol. 2000;37:660-4.

37. Steinhoff C, Franke KH, Golka K, Thier R, Romer HC, Rotzel C, Ackermann R, Schulz WA. Glutathione transferase isozyme genotypes in patients with prostate and bladder carcinoma. Arch Toxicol. 2000;74:521-6.

38. Kim WJ, Lee HL, Lee SC, Kim YT, Kim H. Polymorphisms of Nacetyltransferase 2, glutathione S-transferase mu and theta genes as risk factors of bladder cancer in relation to asthma and tuberculosis. J Urol. 2000;164:209-13.

39. Schnakenberg E, Lustig M, Breuer R, Werdin R, Hubotter R, Dreikorn K, Schloot W. Gender-specific effects of NAT2 and GSTM1 in bladder cancer. Clin Genet. 2000;57:270-7.

40. Peluso M, Airoldi L, Magagnotti C, Fiorini L, Munnia A, Hautefeuille A, Malaveille $C$, Vineis $P$. White blood cell DNA adducts and fruit and vegetable consumption in bladder cancer. Carcinogenesis. 2000;21:183-7.

41. Aktas D, Ozen H, Atsu N, Tekin A, Sozen S, Tuncbilek E. Glutathione Stransferase $\mathrm{M} 1$ gene polymorphism in bladder cancer patients. A marker for invasive bladder cancer? Cancer Genet Cytogenet. 2001;125:1-4.

42. Toruner GA, Akyerli C, Ucar A, Aki T, Atsu N, Ozen H, Tez M, Cetinkaya M, Ozcelik T. Polymorphisms of glutathione S-transferase genes (GSTM1, GSTP1 and GSTT1) and bladder cancer susceptibility in the Turkish population. Arch Toxicol. 2001;75:459-64.

43. Lee SJ, Cho SH, Park SK, Kim SW, Park MS, Choi HY, Choi JY, Lee SY, Im HJ, Kim JY, Yoon KJ, Choi H, Shin SG, Park TW, Rothman N, Hirvonen A, Kang D. Combined effect of glutathione S-transferase M1 and T1 genotypes on bladder cancer risk. Cancer Lett. 2002;177:173-9.

44. Kim WJ, Kim H, Kim CH, Lee MS, Oh BR, Lee HM, Katoh T. GSTT1-null genotype is a protective factor against bladder cancer. Urology. 2002;60: 913-8.

45. Giannakopoulos X, Charalabopoulos K, Baltogiannis D, Chatzikiriakidou A, Alamanos Y, Georgiou I, Evangelou A, Agnantis N, Sofikitis N. The role of Nacetyltransferase-2 and glutathione S-transferase on the risk and aggressiveness of bladder cancer. Anticancer Res. 2002;22:3801-4.

46. Schroeder JC, Conway K, Li Y, Mistry K, Bell DA, Taylor JA. p53 mutations in bladder cancer: evidence for exogenous versus endogenous risk factors. Cancer Res. 2003;63:7530-8.

47. Hung RJ, Boffetta P, Brennan P, Malaveille C, Hautefeuille A, Donato F, Gelatti U, Spaliviero M, Placidi D, Carta A, Scotto di Carlo A, Porru S. GST, NAT, SULT1A1, CYP1B1 genetic polymorphisms, interactions with environmental exposures and bladder cancer risk in a high-risk population. Int J Cancer. 2004;110:598-604.

48. Srivastava DS, Kumar A, Mittal B, Mittal RD. Polymorphism of GSTM1 and GSTT1 genes in bladder cancer: a study from North India. Arch Toxicol. 2004;78:430-4.

49. Kim EJ, Jeong P, Quan C, Kim J, Bae SC, Yoon SJ, Kang JW, Lee SC, Jun Wee J, Kim WJ. Genotypes of TNF-alpha, VEGF, hOGG1, GSTM1, and GSTT1: useful determinants for clinical outcome of bladder cancer. Urology. 2005;65:70-5.

50. Karagas MR, Park S, Warren A, Hamilton J, Nelson HH, Mott LA, Kelsey KT. Gender, smoking, glutathione-S-transferase variants and bladder cancer incidence: a population-based study. Cancer Lett. 2005;219:63-9.

51. Ouerhani S, Tebourski F, Slama MR, Marrakchi R, Rabeh M, Hassine LB, Ayed $M$, Elgaaied $A B$. The role of glutathione transferases $M 1$ and $T 1$ in individual susceptibility to bladder cancer in a Tunisian population. Ann Hum Biol. 2006:33:529-35.

52. Srivastava DS, Mishra DK, Mandhani A, Mittal B, Kumar A, Mittal RD. Association of genetic polymorphism of glutathione S-transferase M1, T1, P1 and susceptibility to bladder cancer. Eur Urol. 2005;48:339-44.

53. Yuan JM, Chan KK, Coetzee GA, Castelao JE, Watson MA, Bell DA, Wang R, Yu MC. Genetic determinants in the metabolism of bladder carcinogens in relation to risk of bladder cancer. Carcinogenesis. 2008;29:1386-93.

54. Shao J, Gu M, Zhang Z, Xu Z, Hu Q, Qian L. Genetic variants of the cytochrome P450 and glutathione S-transferase associated with risk of bladder cancer in a south-eastern Chinese population. Int J Urol. 2008;15: 216-21.

55. Salinas-Sanchez AS, Sanchez-Sanchez F, Donate-Moreno MJ, Rubio-delCampo A, Gimenez-Bachs JM, Lorenzo-Romero JG, Serrano-Oviedo L, Escribano J. Polymorphic deletions of the GSTT1 and GSTM1 genes and susceptibility to bladder cancer. BJU Int. 2011;107:1825-32.

56. Moore LE, Baris DR, Figueroa JD, Garcia-Closas M, Karagas MR, Schwenn MR, Johnson AT, Lubin JH, Hein DW, Dagnall CL, Colt JS, Kida M, Jones MA, Schned AR, Cherala SS, Chanock SJ, Cantor KP, Silverman DT, Rothman N. GSTM1 null and NAT2 slow acetylation genotypes, smoking intensity and bladder cancer risk: results from the New England bladder cancer study and NAT2 meta-analysis. Carcinogenesis. 2011;32:182-9.

57. Goerlitz D, El Daly M, Abdel-Hamid M, Saleh DA, Goldman L, El Kafrawy S, Hifnawy T, Ezzat S, Abdel-Aziz MA, Zaghloul MS, Ali SR, Khaled H, Amr S, Zheng YL, Mikhail N, Loffredo C. GSTM1, GSTT1 null variants, and GPX1 single nucleotide polymorphism are not associated with bladder cancer risk in Egypt. Cancer Epidemiol Biomark Prev. 2011;20:1552-4.

58. Safarinejad MR, Safarinejad S, Shafiei N. Association of genetic polymorphism of glutathione S-transferase (GSTM1, GSTT1, GSTP1) with bladder cancer susceptibility. Urol Oncol. 2013;31:1193-203.

59. Ali SH, Bangash KS, Rauf A, Younis M, Anwar K, Khurram R, Khawaja MA, Azam M, Qureshi AA, Akhter S, Kiemeney LA, Qamar R. Identification of novel potential genetic predictors of urothelial bladder carcinoma susceptibility in Pakistani population. Fam Cancer. 2017;16:577-94.

60. Elhawary NA, Nassir A, Saada H, Dannoun A, Qoqandi O, Alsharif A, Tayeb MT. Combined genetic biomarkers confer susceptibility to risk of urothelial bladder carcinoma in a Saudi population. Dis Markers. 2017;2017:1474560.

61. Garcia-Closas M, Malats N, Silverman D, Dosemeci M, Kogevinas M, Hein DW, Tardon A, Serra C, Carrato A, Garcia-Closas R, Lloreta J, Castano-Vinyals 
G, Yeager M, Welch R, Chanock S, Chatterjee N, Wacholder S, Samanic C, Tora M, Fernandez F, Real FX, Rothman N. NAT2 slow acetylation, GSTM1 null genotype, and risk of bladder cancer: results from the Spanish bladder Cancer study and meta-analyses. Lancet. 2005;366:649-59.

62. Anwar WA, Abdel-Rahman SZ, El-Zein RA, Mostafa HM, Au WW. Genetic polymorphism of GSTM1, CYP2E1 and CYP2D6 in Egyptian bladder cancer patients. Carcinogenesis. 1996;17:1923-9.

63. Ma QW, Lin GF, Chen JG, Shen JH. Polymorphism of glutathione Stransferase T1, M1 and P1 genes in a Shanghai population: patients with occupational or non-occupational bladder cancer. Biomed Environ Sci. 2002; 15:253-60.

64. Jong Jeong H, Jin Kim H, Young Seo I, Ju Kim H, Oh GJ, Cheon Chae S, Sik Lim J, Taeg Chung H, Joong KJ. Association between glutathione Stransferase M1 and T1 polymorphisms and increased risk for bladder cancer in Korean smokers. Cancer Lett. 2003;202:193-9.

65. Moore LE, Wiencke JK, Bates MN, Zheng S, Rey OA, Smith AH. Investigation of genetic polymorphisms and smoking in a bladder cancer case-control study in Argentina. Cancer Lett. 2004;211:199-207.

66. Saad AA, O'Connor PJ, Mostafa MH, Metwalli NE, Cooper DP, Povey AC, Margison GP. Glutathione S-transferase M1, T1 and P1 polymorphisms and bladder cancer risk in Egyptians. Int J Biol Markers. 2005;20:69-72.

67. McGrath M, Michaud D, De Vivo I. Polymorphisms in GSTT1, GSTM1, NAT1 and NAT2 genes and bladder cancer risk in men and women. BMC Cancer. 2006;6:239.

68. Sobti RC, Al-Badran Al, Sharma S, Sharma SK, Krishan A, Mohan H. Genetic polymorphisms of CYP2D6, GSTM1, and GSTT1 genes and bladder cancer risk in North India. Cancer Genet Cytogenet. 2005;156:68-73.

69. Cengiz M, Ozaydin A, Ozkilic AC, Dedekarginoglu G. The investigation of GSTT1, GSTM1 and SOD polymorphism in bladder cancer patients. Int Urol Nephrol. 2007;39:1043-8.

70. Murta-Nascimento C, Silverman DT, Kogevinas M, Garcia-Closas M, Rothman N, Tardon A, Garcia-Closas R, Serra C, Carrato A, Villanueva C, Dosemeci M, Real FX, Malats N. Risk of bladder cancer associated with family history of cancer: do low-penetrance polymorphisms account for the increase in risk? Cancer Epidemiol Biomark Prev. 2007;16:1595-600.

71. Moore LE, Malats N, Rothman N, Real FX, Kogevinas M, Karami S, GarciaClosas R, Silverman D, Chanock S, Welch R, Tardon A, Serra C, Carrato A, Dosemeci M, Garcia-Closas M. Polymorphisms in one-carbon metabolism and trans-sulfuration pathway genes and susceptibility to bladder cancer. Int J Cancer. 2007;120:2452-8.

72. Kellen E, Zeegers M, Paulussen A, Vlietinck R, Vlem EV, Veulemans $H$, Buntinx F. Does occupational exposure to PAHs, diesel and aromatic amines interact with smoking and metabolic genetic polymorphisms to increase the risk on bladder cancer?; the Belgian case control study on bladder cancer risk. Cancer Lett. 2007;245:51-60.

73. Zhao H, Lin J, Grossman HB, Hernandez LM, Dinney CP, Wu X. Dietary isothiocyanates, GSTM1, GSTT1, NAT2 polymorphisms and bladder cancer risk. Int J Cancer. 2007;120:2208-13.

74. Covolo L, Placidi D, Gelatti U, Carta A, Scotto Di Carlo A, Lodetti P, Picciche A, Orizio G, Campagna M, Arici C, Porru S. Bladder cancer, GSTs, NAT1, NAT2, SULT1A1, XRCC1, XRCC3, XPD genetic polymorphisms and coffee consumption: a case-control study. Eur J Epidemiol. 2008;23:355-62.

75. Golka K, Schmidt T, Seidel T, Dietrich H, Roemer HC, Lohlein D, Reckwitz T, Sokeland J, Weistenhofer W, Blaszkewicz M, Selinski S. The influence of polymorphisms of glutathione $\mathrm{S}$-transferases $\mathrm{M} 1$ and $\mathrm{M} 3$ on the development of human urothelial cancer. J Toxicol Environ Health A. 2008; 71:881-6.

76. Altayli E, Gunes S, Yilmaz AF, Goktas S, Bek Y. CYP1A2, CYP2D6, GSTM1, GSTP1, and GSTT1 gene polymorphisms in patients with bladder cancer in a Turkish population. Int Urol Nephrol. 2009;41:259-66.

77. Grando JP, Kuasne H, Losi-Guembarovski R, Sant'ana Rodrigues I, Matsuda HM, Fuganti PE, Gregorio EP, Junior FL, de Menezes RP, de Freitas Rodrigues MA, de Syllos Colus IM. Association between polymorphisms in the biometabolism genes CYP1A1, GSTM1, GSTT1 and GSTP1 in bladder cancer. Clin Exp Med. 2009;9:21-8.

78. Lin J, Kamat A, Gu J, Chen M, Dinney CP, Forman MR, Wu X. Dietary intake of vegetables and fruits and the modification effects of GSTM1 and NAT2 genotypes on bladder cancer risk. Cancer Epidemiol Biomark Prev. 2009;18: 2090-7

79. Song DK, Xing DL, Zhang LR, Li ZX, Liu J, Qiao BP. Association of NAT2, GSTM1, GSTT1, CYP2A6, and CYP2A13 gene polymorphisms with susceptibility and clinicopathologic characteristics of bladder cancer in Central China. Cancer Detect Prev. 2009:32:416-23.

80. Zupa A, Sgambato A, Bianchino G, Improta G, Grieco V, LAT G, Campisi B, Traficante A, Aieta M, Cittadini A. GSTM1 and NAT2 polymorphisms and colon, lung and bladder cancer risk: a case-control study. Anticancer Res. 2009:29:1709-14.

81. Abd El Hameed AH, Negm OE, El-Gamal OM, Hamouda HE, El Nouby KA, Ismail GM. Genetic polymorphism of glutathione S-transferases M1 and T1 in Egyptian patients with bilharzial bladder cancer. Urol Oncol. 2010;28:296-301.

82. Ozturk T, Kahraman OT, Toptas B, Kisakesen HI, Cakalir C, Verim L, Ozturk O, Isbir T. The effect of CYP1A1 and GSTM1 gene polymorphisms in bladder cancer development in a Turkish population. In Vivo. 2011;25:663-8.

83. Rouissi K, Ouerhani S, Hamrita B, Bougatef K, Marrakchi R, Cherif M, Ben Slama MR, Bouzouita M, Chebil M, Ben Ammar Elgaaied A. Smoking and polymorphisms in xenobiotic metabolism and DNA repair genes are additive risk factors affecting bladder cancer in Northern Tunisia. Pathol Oncol Res. 2011;17:879-86.

84. Henriquez-Hernandez LA, Navarro P, Luzardo OP, Alvarez-Leon EE, Boada LD, Zumbado M, Pestano J, Suarez JR, Chesa N, Almeida M, Valeron PF. Polymorphisms of glutathione S-transferase mu and theta, MDR1 and VEGF genes as risk factors of bladder cancer: a case-control study. Urol Oncol. 2012;30:660-5.

85. Ovsiannikov D, Selinski S, Lehmann ML, Blaszkewicz M, Moormann O, Haenel MW, Hengstler JG, Golka K. Polymorphic enzymes, urinary bladder cancer risk, and structural change in the local industry. J Toxicol Environ Health A. 2012;75:557-65.

86. Lesseur C, Gilbert-Diamond D, Andrew AS, Ekstrom RM, Li Z, Kelsey KT, Marsit CJ, Karagas MR. A case-control study of polymorphisms in xenobiotic and arsenic metabolism genes and arsenic-related bladder cancer in New Hampshire. Toxicol Lett. 2012;210:100-6.

87. Schwender H, Selinski S, Blaszkewicz M, Marchan R, Ickstadt K, Golka K, Hengstler JG. Distinct SNP combinations confer susceptibility to urinary bladder cancer in smokers and non-smokers. PLoS One. 2012;7:e51880.

88. Zhang X, Lin J, Wu X, Lin Z, Ning B, Kadlubar S, Kadlubar FF. Association between GSTM1 copy number, promoter variants and susceptibility to urinary bladder cancer. Int J Mol Epidemiol Genet. 2012;3:228-36.

89. Berber U, Yilmaz I, Yilmaz O, Haholu A, Kucukodaci Z, Ates F, Demirel D. CYP1A1 (lle462Val), CYP1B1 (Ala119Ser and Val432Leu), GSTM1 (null), and GSTT1 (null) polymorphisms and bladder cancer risk in a Turkish population. Asian Pac J Cancer Prev. 2013;14:3925-9.

90. Marenne G, Real FX, Rothman N, Rodriguez-Santiago B, Perez-Jurado L, Kogevinas M, Garcia-Closas M, Silverman DT, Chanock SJ, Genin E, Malats N. Genome-wide CNV analysis replicates the association between GSTM1 deletion and bladder cancer: a support for using continuous measurement from SNP-array data. BMC Genomics. 2012;13:326.

91. Kang HW, Song PH, Ha YS, Kim WT, Kim YJ, Yun SJ, Lee SC, Choi YH, Moon SK, Kim WJ. Glutathione S-transferase M1 and T1 polymorphisms: susceptibility and outcomes in muscle invasive bladder cancer patients. Eur J Cancer. 2013:49:3010-9.

92. Matic M, Pekmezovic T, Djukic T, Mimic-Oka J, Dragicevic D, Krivic B, Suvakov S, Savic-Radojevic A, Pljesa-Ercegovac M, Tulic C, Coric V, Simic T. GSTA1, GSTM1, GSTP1, and GSTT1 polymorphisms and susceptibility to smoking-related bladder cancer: a case-control study. Urol Oncol. 2013;31:1184-92.

93. Savic-Radojevic A, Djukic T, Simic T, Pljesa-Ercegovac M, Dragicevic D, Pekmezovic T, Cekerevac M, Santric V, Matic M. GSTM1-null and GSTA1-low activity genotypes are associated with enhanced oxidative damage in bladder cancer. Redox Rep. 2013;18:1-7.

94. Wang Z, Xue L, Chong T, Li H, Chen H. Quantitative assessment of the association between glutathione S-transferase P1 lle105Val polymorphism and bladder cancer risk. Tumour Biol. 2013;34:1651-7.

95. Reszka E, Jablonowski Z, Wieczorek E, Jablonska E, Krol MB, Gromadzinska J, Grzegorczyk A, Sosnowski M, Wasowicz W. Polymorphisms of NRF2 and NRF2 target genes in urinary bladder cancer patients. J Cancer Res Clin Oncol. 2014;140:1723-31.

96. Ceylan GG, Ceylan C, Tasdemir S, Gozalan A. The effect of glutathione-Stransferases in the susceptibility to bladder cancer. Ir J Med Sci. 2015;184:851-4.

97. Chen YC, Xu L, Guo YL, Su HJ, Smith TJ, Ryan LM, Lee MS, Christiani DC Polymorphisms in GSTT1 and p53 and urinary transitional cell carcinoma in South-Western Taiwan: a preliminary study. Biomarkers. 2004;9:386-94.

98. Kempkes M, Golka K, Reich S, Reckwitz T, Bolt HM. Glutathione S-transferase GSTM1 and GSTT1 null genotypes as potential risk factors for urothelial cancer of the bladder. Arch Toxicol. 1996;71:123-6. 
99. Kim H, Kim W, Lee H. A case-control study on the effects of the genetic polymorphisms of $\mathrm{N}$-acetyltransferase 2 and glutathione Stransferase mu and theta on the risk of bladder cancer. Korean J Prevent Med. 1998;31:275-84.

100. Lee S, Kang D, Cho S. Association of genetic polymorphism of glutathione s-transferase M1 and T1 and bladder cancer. J Korean Cancer Assoc. 1999; 31:548-55.

101. Gago-Dominguez M, Bell DA, Watson MA, Yuan JM, Castelao JE, Hein DW, Chan KK, Coetzee GA, Ross RK, Yu MC. Permanent hair dyes and bladder cancer: risk modification by cytochrome P4501A2 and N-acetyltransferases 1 and 2. Carcinogenesis. 2003;24:483-9.

102. Sanyal S, Festa F, Sakano S, Zhang Z, Steineck G, Norming U, Wijkstrom H, Larsson P, Kumar R, Hemminki K. Polymorphisms in DNA repair and metabolic genes in bladder cancer. Carcinogenesis. 2004;25:729-34.

103. Broberg K, Bjork J, Paulsson K, Hoglund M, Albin M. Constitutional short telomeres are strong genetic susceptibility markers for bladder cancer. Carcinogenesis. 2005;26:1263-71.

104. Golka K, Seidel T, Dietrich H, Roth G, Rotzel C, Thier R, Geller F, Reckwitz T, Schulze $\mathrm{H}$. Occupational and non-occupational risk factors in bladder cancer patients in an industrialized area located in former East-Germany. Aktuelle Urol. 2005;36:417-22.

105. Shao C, Xiang Y, Zhang W. Polymorphisms of GSTM1 and GSTT1 with smoking and bladder cancer risk: a population-based case control study. Tumori. 2006;26:346-51.

106. Kogevinas M, Fernandez F, Garcia-Closas M, Tardon A, Garcia-Closas R, Serra C, Carrato A, Castano-Vinyals G, Yeager M, Chanock SJ, Lloreta J, Rothman N, Real FX, Dosemeci M, Malats N, Silverman D. Hair dye use is not associated with risk for bladder cancer: evidence from a case-control study in Spain. Eur J Cancer. 2006;42:1448-54.

107. Song D, Xing D, Zhang L. Relationship between polymorphism of glutathione stransferase and genetic susceptibility to bladder cancer. Chin J Urol. 2008;29:80-3.

108. Cantor KP, Villanueva CM, Silverman DT, Figueroa JD, Real FX, Garcia-Closas M, Malats N, Chanock S, Yeager M, Tardon A, Garcia-Closas R, Serra C, Carrato A, Castano-Vinyals G, Samanic C, Rothman N, Kogevinas M. Polymorphisms in GSTT1, GSTZ1, and CYP2E1, disinfection by-products, and risk of bladder cancer in Spain. Environ Health Perspect. 2010;118:1545-50.

109. Wu MY, Huang SJ, Yang F, Qin XT, Liu D, Ding Y, Yang S, Wang XC Detection of nasopharyngeal carcinoma susceptibility with single nucleotide polymorphism analysis using next-generation sequencing technology. Oncotarget. 2017;8:52708-23.

110. Engel LS, Taioli E, Pfeiffer R, Garcia-Closas M, Marcus PM, Lan Q, Boffetta P, Vineis $P$, Autrup H, Bell DA, Branch RA, Brockmoller J, Daly AK, Heckbert SR Kalina I, Kang D, Katoh T, Lafuente A, Lin HJ, Romkes M, Taylor JA, Rothman N. Pooled analysis and meta-analysis of glutathione S-transferase M1 and bladder cancer: a HuGE review. Am J Epidemiol. 2002;156:95-109.

111. Yu C, Hequn C, Longfei L, Long W, Zhi C, Feng Z, Jinbo C, Chao L, Xiongbing Z. GSTM1 and GSTT1 polymorphisms are associated with increased bladder cancer risk: evidence from updated meta-analysis, Oncotarget. 2017;8:3246-58.

112. Yu Y, Li X, Liang C, Tang J, Qin Z, Wang C, Xu W, Hua Y, Shao P, Xu T. The relationship between GSTA1, GSTM1, GSTP1, and GSTT1 genetic polymorphisms and bladder cancer susceptibility: A meta-analysis. Medicine (Baltimore). 2016;95:e4900

113. Fu D, Li P, Cheng W, Tian F, Xu X, Yi X, Tang C, Wang Y, Hu Q, Zhang Z. Impact of vascular endothelial growth factor gene-gene and gene-smoking interaction and haplotype combination on bladder cancer risk in Chinese population. Oncotarget. 2017:8:22927-35.

114. Lacombe L, Fradet V, Levesque E, Pouliot F, Larue H, Bergeron A, Hovington H, Caron A, Nguile-Makao M, Harvey M, Fradet Y, Guillemette C. Phase II drug-metabolizing polymorphisms and smoking predict recurrence of nonmuscle-invasive bladder Cancer: a gene-smoking interaction. Cancer Prev Res (Phila). 2016;9:189-95.

Ready to submit your research? Choose BMC and benefit from:

- fast, convenient online submission

- thorough peer review by experienced researchers in your field

- rapid publication on acceptance

- support for research data, including large and complex data types

- gold Open Access which fosters wider collaboration and increased citations

- maximum visibility for your research: over $100 \mathrm{M}$ website views per year

At $\mathrm{BMC}$, research is always in progress.

Learn more biomedcentral.com/submissions 